\title{
Experimental evaluation of two complementary decentralized event-based control methods
}

\author{
M. Sigurani ${ }^{\mathrm{a}}$, C. Stöcker ${ }^{\mathrm{b}, *}$, L. Grüne ${ }^{\mathrm{a}}$, J. Lunze ${ }^{\mathrm{b}}$ \\ ${ }^{a}$ University of Bayreuth, Mathematical Institute, Germany \\ ${ }^{b}$ Ruhr-University Bochum, Institute of Automation and Computer Control, Germany
}

\begin{abstract}
Event-based control aims at reducing the feedback communication effort among the sensors, controllers and actuators in control loops to time instants at which the feedback of information is necessary to meet a desired control performance. This paper presents a new method for the decentralized event-based control of physically interconnected systems and shows its experimental evaluation. The novel method is based on two complementary approaches, called the global and the local approach, which jointly ensure the ultimate boundedness of the closed-loop system. The global approach steers the state of each subsystem into a target region, whereas the local approach keeps the state in this set in spite of exogenous disturbances and the effect of the interconnections to other subsystems. This event-based control method is applied to a continuous flow process to show its practical implementation and to evaluate the analytical results on the basis of experiments.
\end{abstract}

Keywords: Event-based control, Ultimate boundedness, Networked control system, Interconnected systems, Thermofluid Process

\section{Introduction}

In event-based control the communication among the components of a control system is restricted to time instants at which the exchange of current information is necessary to ensure a desired behavior of the closed-loop system. Its triggering scheme contrasts with the current practice, where the controller is implemented on digital hardware and the control task is executed periodically (sampled-data control). As the main reason for using this kind of implementation, the analysis and design of sampled-data control loops can be based on a well-established theory. However, the periodic sampling, computing and updating of actuator signals is carried out whether required or not, which can lead to a waste of communication resources. To use these communication resources more efficiently, eventbased control has been proposed as an alternative to periodic control (see Heemels et al. (2012)).

This paper investigates decentralized event-based control of interconnected systems $\Sigma_{i}(i=1, \ldots, N)$ (Fig. 1). The eventbased controller for each subsystem $\Sigma_{i}$ consists of an event generator $E_{i}$ and a control input generator $C_{i}$ which communicate over a network only at certain event times. The aim of this paper is twofold: First, it is presented how two approaches to decentralized event-based control can be combined to jointly accomplish ultimate boundedness of the overall control system. These two approaches, which are subsequently specified as the global approach and the local approach to decentralized event-based control, are based on methods which have been recently published by Grüne \& Sigurani (2013) and Stöcker et al. (2013),

\footnotetext{
* Corresponding author

Email addresses: manuela.sigurani@uni-bayreuth.de (M. Sigurani), stoecker@atp.rub.de (C. Stöcker),

lars.gruene@uni-bayreuth.de (L. Grüne), lunze@atp.rub.de (J. Lunze)
}

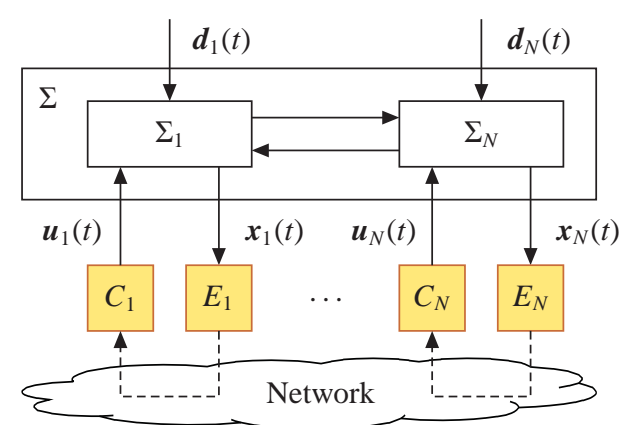

Figure 1: Structure of the event-based control system

respectively. The global approach drives the state of each subsystem into a target region in finite time where the local approach takes over and keeps the state within this region in spite of exogenous disturbances or interconnections to other systems. The second aim of this paper is to demonstrate how these complementary approaches are applied in practice to a continuous flow process. The analytical results derived in the first part of this paper are evaluated by experiments.

Event-based control is a useful means to reduce the communication while accomplishing a desired control performance which has been shown in several simulation and experimental studies, e.g. by Hendricks et al. (1994); Heemels et al. (1999); Kwon et al. (1999); Yook et al. (2002); Sandee et al. (2007); Henningsson \& Cervin (2009); Lehmann \& Lunze (2011); Trimpe \& D'Andrea (2011). Besides the investigation of the practical applicability, a lot of effort has been spend on developing a profound theory on event-based control starting with the works of Arzén (1999); Åström \& Bernhardsson (1999) and has been continued in recent years, e.g. by Tabuada (2007); Henningsson et al. (2008); Gawthrop \& Wang (2009); 
Lunze \& Lehmann (2010); Yu \& Antsaklis (2011); Donkers \& Heemels (2012); Wang \& Lemmon (2012); Molin \& Hirche (2013). Most of the literature on event-based control is concerned with stabilization. The problem of rendering the system asymptotically or exponentially stable using event-based feedback has been studied, among others, by Tabuada (2007); Mazo \& Tabuada (2010); De Persis et al. (2011); Garcia \& Antsaklis (2011); Wang \& Lemmon (2012). Except for the works of Wang \& Lemmon (2011a) and Stöcker \& Lunze (2013), the price for asymptotic stability is usually a more frequent communication the closer the state converges to the desired target point. Moreover, the event-based control approaches which achieve asymptotic stability do not tolerate model uncertainties or exogenous disturbances.

From a practical perspective it is more preferable to steer the system state into a target region, rather than a point, and maintain it there, which is known in literature as ultimate boundedness (see e.g. Khalil (2002)). Event-based control approaches which aim at this property have been presented by Lunze \& Lehmann (2010); Grüne et al. (2010); Lehmann (2011); Tallapragada \& Chopra (2011); Wang \& Lemmon (2011b); Donkers \& Heemels (2012). According to the definition of ultimate boundedness the state must enter the target set within some finite time $T$ and remain there for all $t \geq T$. However, in none of the publications that study ultimate boundedness of event-based control systems (Grüne et al. (2010); Lehmann (2011); Tallapragada \& Chopra (2011)), the time $T$ (or a bound on it) is derived. In contrast, this paper gives an upper bound on the time $T$ in which the state attains the target set.

While early works on event-based control have been mainly focused on single-loop systems, some recent publications deal with decentralized control (Mazo \& Tabuada (2010); Stöcker et al. (2013); Garcia \& Antsaklis (2012)) or distributed control (Wang \& Lemmon (2011b); De Persis et al. (2011)). In the existing literature the plant is considered to be exclusively described by either nonlinear dynamics (Tabuada (2007); Mazo \& Tabuada (2010); De Persis et al. (2011); Stöcker \& Lunze (2011); Wang \& Lemmon (2012) Grüne \& Müller (2009)) or by linear dynamics (Lunze \& Lehmann (2010); Donkers \& Heemels (2012)). The approach presented in this paper uses both a nonlinear model for the global approach and a linearized model for the local approach. In this way, this paper follows an idea that has been published by Grüne et al. (2010) for singleloop systems and extends it to decentralized control. Based on the separation of the control problem (ultimate boundedness of the closed-loop system) into two problems, namely

1. steering the state from an initial state into a target region and

2. keeping the state in the target region in spite of exogenous disturbances or interconnections with other subsystems,

the global and the local approach are tailored to the respective task. The combination of both approaches leads to a new method for the decentralized stabilization and disturbance attenuation of interconnected systems. Although the global and the local approach use different models (nonlinear vs. linear) and follow different ideas (stabilization vs. disturbance attenuation), they are similar in the sense that event-based controllers are designed for the isolated subsystems and their robustness with respect to the interactions of the subsystems are proved by small-gain theorems. The main contribution of this paper is the experimental evaluation of the combination of the global and the local event-based control approach on a thermofluid process that is realized in bench scale using standard industrial components.

Outline of the paper. The general control problem is formally stated in Sec. 2. The global approach and the local approach are explained in Sections 3 and 4, respectively. The combination of both control methods is described in Sec. 5. Sections 6 introduces the continuous flow process, explains the implementation of the decentralized event-based controllers and discusses the main differences between the theoretical foundation and the practical realization. The experimental results are presented in Sec. 7

Notation. $\mathbb{R}$ and $\mathbb{R}_{+}$denote the set of real numbers and nonnegative real numbers, respectively, and $\bar{B}_{1}(0)$ denotes the closed unit ball. For a scalar $s,|s|$ denotes the absolute value. For a vector $\boldsymbol{v} \in \mathbb{R}^{n}$ or a matrix $\boldsymbol{M} \in \mathbb{R}^{m \times n}$ the $|\cdot|$-operator applies to every element. A comparison between two vectors $\boldsymbol{v}, \tilde{\boldsymbol{v}} \in \mathbb{R}^{n}$ holds element-wise, like $\boldsymbol{v} \leq \tilde{\boldsymbol{v}}$ amounts to $v_{i} \leq \tilde{v}_{i}$ for all $i=1, \ldots, n$, where $v_{i}$ and $\tilde{v}_{i}$ denote the $i$ th element of the vectors $\boldsymbol{v}$ and $\tilde{\boldsymbol{v}}$, respectively. For a real square matrix $\boldsymbol{M} \in \mathbb{R}^{n \times n}, \lambda_{\mathrm{P}}(\boldsymbol{M})$ denotes its Perron root (largest eigenvalue of $\boldsymbol{M}$ ). A block diagonal matrix with the matrices $\boldsymbol{A}_{i}$ for $i=1, \ldots, N$ on the main diagonal is represented by $\boldsymbol{A}=\operatorname{diag}\left(\boldsymbol{A}_{i}\right)$. For a signal $\boldsymbol{x}: \mathbb{R}_{+} \rightarrow \mathbb{R}^{n}$, the limit from above at time $t \in \mathbb{R}_{+}$is denoted by $\boldsymbol{x}\left(t^{+}\right)=\lim _{s \downarrow t} \boldsymbol{x}(s)$. We introduce the following sets of comparison functions: $\mathcal{K}=\{\gamma$ : $\mathbb{R}_{+} \rightarrow \mathbb{R}_{+} \mid \gamma$ is continuous, strictly increasing, and $\left.\gamma(0)=0\right\}$, $\mathcal{K}_{\infty}=\{\gamma \in \mathcal{K} \mid \gamma$ is unbounded $\}$ and $\mathcal{K} \mathcal{L}=\left\{\beta: \mathbb{R}_{+} \times \mathbb{R}_{+} \rightarrow\right.$ $\mathbb{R}_{+} \mid \beta(s, t)$ is continuous, $\beta(\cdot, t) \in \mathcal{K}$ and for each fixed $s \geq 0$ the function $\beta(s, t)$ is decreasing to zero as $t \rightarrow \infty$.

\section{Problem formulation}

\subsection{Structure of the event-based control system}

This paper investigates decentralized event-based control of physically interconnected systems. The considered structure of the event-based control system is illustrated in Fig. 1. The overall plant $\Sigma$ is composed of $N$ physically interconnected subsystems $\Sigma_{i},(i=1, \ldots, N)$. The subsystem $\Sigma_{i}$ is controlled by an event-based controller consisting of the control input generator $C_{i}$ and the event generator $E_{i}$.

- The event generator $E_{i}$ determines the event times $t_{k}$, at which a feedback communication is invoked, based on locally available information only and

- the control input generator $C_{i}$ produces the control input for subsystem $\Sigma_{i}$ using only information received at the event times from the event generator $E_{i}$. 
The dashed lines in Fig. 1 represent a feedback communication from $E_{i}$ to $C_{i}$ that occurs at the event times $t_{k}$ only, whereas the solid lines represent continuous information links. In this paper, the information transmission over the communication network is assumed to occur instantaneously and without delays or packet losses.

\subsection{Plant model}

The overall plant is composed of $N$ subsystems and is described by the nonlinear state-space model

$$
\Sigma:\left\{\begin{array}{c}
\dot{\boldsymbol{x}}_{1}(t)=\boldsymbol{h}_{1}\left(\boldsymbol{x}_{1}(t), \ldots, \boldsymbol{x}_{N}(t), \boldsymbol{u}_{1}(t), \boldsymbol{d}_{1}(t)\right) \\
\vdots \\
\dot{\boldsymbol{x}}_{N}(t)=\boldsymbol{h}_{N}\left(\boldsymbol{x}_{1}(t), \ldots, \boldsymbol{x}_{N}(t), \boldsymbol{u}_{N}(t), \boldsymbol{d}_{N}(t)\right)
\end{array}\right.
$$

with initial conditions $\boldsymbol{x}_{i}(0)=\boldsymbol{x}_{0 i}$ for all $i \in \mathcal{N}:=\{1, \ldots, N\}$. In (1), $\boldsymbol{x}_{i} \in \mathcal{X}_{i} \subset \mathbb{R}^{n_{i}}, \boldsymbol{u}_{i} \in \mathcal{U}_{i} \subset \mathbb{R}^{m_{i}}$ and $\boldsymbol{d}_{i} \in \mathcal{D}_{i} \subset \mathbb{R}^{w_{i}}$ are the state, the control input and the disturbance of the $i$-th subsystem denoted by $\Sigma_{i}$. In the following,

$$
\mathcal{D}_{i}:=\left\{\boldsymbol{d}_{i} \in \mathbb{R}^{w_{i}}|| \boldsymbol{d}_{i} \mid \leq \overline{\boldsymbol{d}}_{i}\right\}
$$

holds, where the vector $\overline{\boldsymbol{d}}_{i}$ element-wise denotes the maximum magnitude of the disturbance vector $\boldsymbol{d}_{i}(t)$. The state, the control input and the disturbance of the overall plant $\Sigma$ are represented by

$$
\begin{aligned}
\boldsymbol{x}(t) & =\left(\boldsymbol{x}_{1}^{\top}(t), \ldots, \boldsymbol{x}_{N}^{\top}(t)\right)^{\top} \\
\boldsymbol{u}(t) & =\left(\boldsymbol{u}_{1}^{\top}(t), \ldots, \boldsymbol{u}_{N}^{\top}(t)\right)^{\top} \\
\boldsymbol{d}(t) & =\left(\boldsymbol{d}_{1}^{\top}(t), \ldots, \boldsymbol{d}_{N}^{\top}(t)\right)^{\top} .
\end{aligned}
$$

The full state space, set of controls and set of disturbances are denoted by $\mathcal{X}=\mathcal{X}_{1} \times \ldots \times \mathcal{X}_{N}, \mathcal{U}=\mathcal{U}_{1} \times \ldots \times \mathcal{U}_{N}$ and $\mathcal{D}=$ $\mathcal{D}_{1} \times \ldots \times \mathcal{D}_{N}$, respectively. Moreover, the set $\mathcal{X}_{i}$ is assumed to be compact for each $i \in \mathcal{N}$.

\subsection{Control aim}

The control aim is formulated in terms of ultimate boundedness:

Definition 1 (Ultimate boundedness, Khalil (2002)). The solution $\boldsymbol{x}(t)$ of (1) is called ultimately bounded $(U B)$ to the set $\mathcal{A}$ if for each initial condition $\boldsymbol{x}_{0} \in \mathcal{X}$ there exists a time $T\left(\boldsymbol{x}_{0}\right)>0$ such that

$$
\boldsymbol{x}(t) \in \mathcal{A}, \quad \forall t \geq T\left(\boldsymbol{x}_{0}\right)
$$

holds for all admissible disturbances $\boldsymbol{d}(t) \in \mathcal{D}$. The system (1) is said to be ultimately bounded if its state $\boldsymbol{x}(t)$ is $U B$.

Consider that the system $\Sigma$ as in (1) and a target set $\mathcal{A}=$ $\mathcal{A}_{1} \times \ldots \times \mathcal{A}_{N}$ are given, where $\mathcal{A}_{i} \subset \mathcal{X}_{i}$ denotes the target set for the subsystem $\Sigma_{i}$. The aim of the first part of this paper is to develop a method for the design of decentralized eventbased state-feedback controllers $\boldsymbol{K}_{i}: \mathcal{X}_{i} \rightarrow \mathcal{U}_{i}$ that render the closed-loop system ultimately bounded to the set $\mathcal{A}$.

\subsection{A global and a local approach}

The proposed event-based control approach follows an idea that has been presented by Grüne et al. (2010) for single-loop event-based control systems. As illustrated in Fig. 2, the problem of finding the decentralized event-based controllers $\boldsymbol{K}_{i}$ that render the closed-loop system ultimately bounded is subdivided into a global problem and a local problem, which are solved by two complementary approaches:

1. The global event-based control approach drives the state $\boldsymbol{x}_{i}(t)$ of each subsystem $\Sigma_{i}$ from the initial state $\boldsymbol{x}_{0 i}$ into the target set $\mathcal{A}_{i}$, while taking possible constraints on the states or on the control inputs into account.

2. The local event-based control approach makes the set $\mathcal{A}_{i}$ for each $\Sigma_{i}$ robustly positive invariant, i.e., once the state $\boldsymbol{x}_{i}(t)$ enters $\mathcal{A}_{i}$ it is kept within this set in spite of exogenous disturbances $\boldsymbol{d}_{i}(t)$ and interconnections to other subsystems.

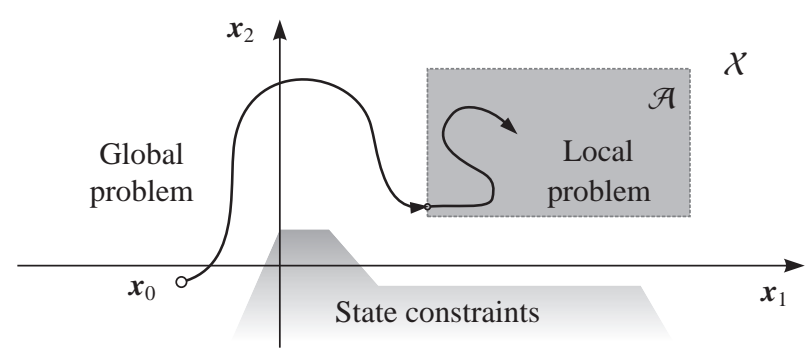

Figure 2: The global and the local problem

Global approach - Nonlinear control. Section 3 proposes an optimization-based method for the design of a decentralized event-based controller which accomplishes the transition of the state $\boldsymbol{x}(t)$ from the initial state $\boldsymbol{x}_{0}$ to the target set $\mathcal{A}$. For the purpose of designing the event-based state-feedback law, this approach uses a nonlinear discrete-time model, obtained by discretizing the plant model given in Eq. (1). For evaluating the control law only quantized information $\left[x_{i}(t)\right]$ about the state $\boldsymbol{x}_{i}(t)$ is applied. The quantization coarsely partitions the state space $X_{i}$ into a grid of boxes in each of which the control input $\boldsymbol{u}_{i}(t)$ is held constant. The event-based character of the controller lies in updating the control input $\boldsymbol{u}_{i}(t)$ only after the state $\boldsymbol{x}_{i}(t)$ has crossed the boundary of a box, which is detected by the event generator $E_{i}$. The control input generator $C_{i}$ is realized as a look-up table that can be computed offline.

Local approach - Linear control. For the local approach, the target set $\mathcal{A}$ is considered as a bounded surrounding of the operating point $\overline{\boldsymbol{x}}$ in which the system $\Sigma$ is described by a linearized model with sufficient accuracy. The linear model that is used for the controller design is obtained from the plant model (1) by means of linearization. The control input generator $C_{i}$ determines the signal $\boldsymbol{u}_{i}(t)$ using a linear model $\Sigma_{\mathrm{s} i}$ of the subsystem $\Sigma_{i}$ with a continuous state-feedback controller. The event generator $E_{i}$ monitors the deviation between the actual subsystem 
state $\boldsymbol{x}_{i}(t)$ and the state $\boldsymbol{x}_{\mathrm{s} i}(t)$ of the model $\Sigma_{\mathrm{s} i}$ used for the generation of the control input $\boldsymbol{u}_{i}(t)$ and it triggers an event whenever this deviation exceeds a defined threshold.

Switching from the global to the local approach. The control input generator $C_{i}$ as well as the event generator $E_{i}$ include the respective components which are designed according to the global and the local approach. $C_{i}$ and $E_{i}$ work as specified by the global approach as long as $\boldsymbol{x}_{i}(t) \notin \mathcal{A}_{i} . T_{i}$ denotes the time instant at which $\boldsymbol{x}_{i}\left(T_{i}\right)$ enters the set $\mathcal{A}_{i}$. At time $t=T_{i}$, both $C_{i}$ and $E_{i}$ switch their functionality and proceed with working according to the local approach and, hence, the state $\boldsymbol{x}_{i}(t)$ is maintained within the set $\mathcal{A}_{i}$ for all $t \geq T_{i}$.

\section{The global approach}

\subsection{Main idea}

In this section the problem of optimally controlling a nonlinear interconnected system to a desired target set $\mathcal{A}$ by means of a quantized state-feedback law is considered. In order to obtain a decentralized controller, we use an input-to-state stability (ISS) based small gain theorem. This approach allows to design the controllers of the subsystems independent of each other by considering the inputs from the other subsystems as perturbations. The individual controllers, in turn, must then be robust w.r.t. these perturbation inputs in the ISS sense. In order to compute these controllers, we show how to convert the ISS design problem into a robust design problem, which can be solved with a set oriented approach presented in Grüne \& Müller (2009), Grüne et al. (2010).

The remainder of this section is devoted to explain some aspects of this approach in more detail. As the set oriented design yields only practical stability, we will utilize practical versions of all stability properties involved. For simplicity of exposition, throughout this section we assume that the target set $\mathcal{A}$ contains a neighborhood of the origin.

Since our approach requires a discrete time model, we sample (1) to obtain a discrete-time representation of the continuous-time sampled-data system

$\Sigma: \boldsymbol{x}(k+1)=\left\{\begin{aligned} \boldsymbol{x}_{1}(k+1) & =\boldsymbol{f}_{1}\left(\boldsymbol{x}_{1}(k), \ldots, \boldsymbol{x}_{N}(k), \boldsymbol{u}_{1}(k), \boldsymbol{d}_{1}(k)\right) \\ & \vdots \\ \boldsymbol{x}_{N}(k+1) & =\boldsymbol{f}_{N}\left(\boldsymbol{x}_{1}(k), \ldots, \boldsymbol{x}_{N}(k), \boldsymbol{u}_{N}(k), \boldsymbol{d}_{N}(k)\right)\end{aligned}\right.$

$$
=\boldsymbol{f}(\boldsymbol{x}(k), \boldsymbol{u}(k), \boldsymbol{d}(k)),
$$

$k=0,1, \ldots$, with initial conditions $\boldsymbol{x}_{i}(0)=\boldsymbol{x}_{0 i}$ for all $i=$ $1, \ldots, N$, where $\boldsymbol{x}_{i} \in \mathcal{X}_{i} \subset \mathbb{R}^{n_{i}}, \boldsymbol{u}_{i} \in \mathcal{U}_{i} \subset \mathbb{R}^{m_{i}}$ and $\boldsymbol{d}_{i} \in \mathcal{D}_{i} \subset$ $\mathbb{R}^{w_{i}}$. Infinite sequences of control and perturbation are denoted by $\underline{\boldsymbol{u}}=(\boldsymbol{u}(0), \boldsymbol{u}(1), \ldots) \in \mathcal{U}^{\mathbb{N}_{0}}$ and $\underline{\boldsymbol{d}}=(\boldsymbol{d}(0), \boldsymbol{d}(1), \ldots) \in \mathcal{D}^{\mathbb{N}_{0}}$.

\subsection{Small gain approach}

We want to construct a decentralized static state feedback controller $\boldsymbol{u}_{\mathcal{P}}$, such that the closed loop system

$$
\boldsymbol{x}(k+1)=\boldsymbol{f}\left(\boldsymbol{x}(k), \boldsymbol{u}_{\mathcal{P}}(\boldsymbol{x}(k)), \underline{\boldsymbol{d}}(k)\right)
$$

is rendered input-to-state practically stable in the following sense.

Definition 2. System (5) is called input-to-state practically stable (ISpS) with respect to $\delta, \Delta_{d} \in \mathbb{R}_{\geq 0}$ on a set $\mathcal{Y} \subset \mathcal{X}$ if there exist $\beta \in \mathcal{K} \mathcal{L}$ and $\gamma \in \mathcal{K}$, such that the solutions of the system satisfy

$$
\|\boldsymbol{x}(k)\| \leq \max \left\{\beta\left(\left\|x_{0}\right\|, k\right), \gamma\left(\|\underline{d}\|_{\infty}\right), \delta\right\},
$$

for all $\boldsymbol{x}_{0} \in \mathcal{Y}$, all $\underline{\boldsymbol{d}} \in \mathcal{D}^{\mathbb{N}_{0}}$ with $\|\underline{\boldsymbol{d}}\|_{\infty} \leq \Delta_{d}$ and all $k \in \mathbb{N}_{0}$.

Since we assumed that the target set $\mathcal{A}$ contains a neighborhood of the origin, this property ensures ultimate boundedness w.r.t. $\mathcal{A}$ provided $\delta$ and $\|\boldsymbol{d}\|_{\infty}$ are sufficiently small.

The idea we pursue for the decentralized design is to derive an ISpS-controller for each subsystem $\Sigma_{i}$, where the input from the other subsystems is considered as an additional perturbation. Then, stability of the overall system can be ensured via a small-gain argument. The central tool for this purpose are ISpS Lyapunov functions since ISpS can be characterized through them. Here we provide the definition of an ISpS Lyapunov function for the subsystems.

Definition 3. A function $V_{i}: \mathcal{X}_{i} \rightarrow \mathbb{R}_{\geq 0}$ is called ISpS Lyapunov function for the $i$-th subsystem $\Sigma_{i}$ of (4) on a sublevel set $\mathcal{Y}_{i}=\left\{\boldsymbol{x}_{i} \in \mathcal{X}_{i} \mid V_{i}\left(\boldsymbol{x}_{i}\right) \leq \ell_{i}\right\}$ for some $\ell_{i}>0$ with respect to a Lyapunov target set $\mathcal{A}_{i}^{V} \subset \mathcal{Y}_{i}$, if there exist functions $\underline{\alpha}_{i}, \bar{\alpha}_{i} \in \mathcal{K}_{\infty}, \alpha_{i}, \mu_{i j} \in \mathcal{K} \cup\{0\}, \mu_{i} \in \mathcal{K}$, a value $\bar{d}_{i} \in \mathbb{R}_{>0}$ such that for all $\boldsymbol{x} \in \mathcal{Y}_{i} \backslash \mathcal{A}_{i}^{V}$ the inequalities and implications

$$
\underline{\alpha}_{i}\left(\left\|\boldsymbol{x}_{i}\right\|\right) \leq V_{i}\left(\boldsymbol{x}_{i}\right) \leq \bar{\alpha}_{i}\left(\left\|\boldsymbol{x}_{i}\right\|\right)
$$

and

$$
\begin{aligned}
V_{i}\left(\boldsymbol{x}_{i}\right) & \left.\geq \max \left\{\max _{j \neq i}\left\{\mu_{i j}\left(V_{j}\left(\boldsymbol{x}_{j}\right)\right)\right\}, \mu_{i}\left(\|\boldsymbol{d}\|_{\infty}\right)\right\}\right\} \\
& \Rightarrow V_{i}\left(\boldsymbol{x}_{i}(k+1)\right)-V_{i}\left(\boldsymbol{x}_{i}(k)\right) \leq-\alpha_{i}\left(\left\|\boldsymbol{x}_{i}\right\|\right)
\end{aligned}
$$

hold for all $\boldsymbol{d}_{i} \in \mathcal{D}_{i}$ with $\left\|\boldsymbol{d}_{i}\right\| \leq \bar{d}_{i}$. The functions $\mu_{i j}, j \neq i$ and $\mu_{i}$ are called Lyapunov gains.

Similarly, we define an ISpS Lyapunov function $V$ for the overall system by omitting all indices $i$ and setting $\mu_{i j} \equiv 0$. The ISS small gain theorem (see, e.g., Dashkovskiy et al. (2010) for continuous time ISS systems and Grüne \& Sigurani (2014) for discrete time ISpS systems) then states that the existence of ISpS Lyapunov functions $V_{i}$ imply the existence of an overall ISpS Lyapunov function $V=\max _{i} \sigma_{i}\left(V_{i}\right)$ for suitable scalar scaling functions $\sigma_{i}$, provided the gains $\mu_{i j}$ are sufficiently small. The resulting Lyapunov target $\mathcal{A}^{V}$ is given as a level set of $V$ and shrinks to 0 if the decentralized targets $\mathcal{A}_{i}^{V}$ shrink to 0 .

The existence of an ISpS Lyapunov function $V$, in turn, implies that the system is ISpS, as the following theorem shows. In order to deal with the inherent discontinuities of our quantized feedback, we assume that there exists a $\gamma_{d} \in \mathcal{K}_{\infty}$, such that for all $\boldsymbol{x} \in \mathcal{X}, \boldsymbol{u} \in \mathcal{U}$ and $\boldsymbol{d} \in \mathcal{D}$ we have $\|\boldsymbol{f}(\boldsymbol{x}, \boldsymbol{u}, \boldsymbol{d})-\boldsymbol{f}(\boldsymbol{x}, \boldsymbol{u}, \mathbf{0})\| \leq$ $\gamma_{d}(\|\boldsymbol{d}\|)$ and that there exists $\gamma_{x} \in \mathcal{K}_{\infty}$ such that for all sufficiently small sets $\mathcal{A} \subset \mathcal{X}$ with $\mathbf{0} \in \mathcal{A}$ and each $\boldsymbol{x} \in \mathcal{A}$ we have $\left\|\boldsymbol{f}\left(\boldsymbol{x}, \boldsymbol{u}_{\mathcal{P}}(\boldsymbol{x}), \boldsymbol{0}\right)\right\| \leq \gamma_{x}(\|\boldsymbol{x}\|)$. Under these assumptions, the following theorem holds, cf. (Grüne \& Sigurani, 2013, Theorem 10). 
Theorem 1. Consider system (5) with $\tilde{f}$ satisfying the two inequalities, above, and assume that the system admits an ISpS Lyapunov function $V$. Then the system is ISpS on $\mathcal{Y}$, with $\Delta_{d} \rightarrow \infty$ as $\ell \rightarrow \infty$ and $\delta \rightarrow 0$ as $\sup _{x \in \mathcal{A}^{V}}\|x\| \rightarrow 0$, provided $\delta \leq \underline{\alpha}^{-1}(\ell)$ holds.

The relation between the size of the Lyapunov target $\mathcal{A}^{V}$ and the practical stability parameter $\delta$ is made more precise in (Grüne $\&$ Sigurani, 2013, Theorem 10). For our purpose, the decisive property is that $\delta \rightarrow 0$ if $\sup _{x \in \mathcal{A}^{V}}\|x\| \rightarrow 0$, which in turn holds if $\sup _{x \in \mathcal{A}_{i}^{V}, i=1, \ldots, N}\|\boldsymbol{x}\| \rightarrow 0$. Since the target $\mathcal{A}$ contains a neighborhood of the origin, this convergence implies ultimate boundedness w.r.t. $\mathcal{A}$ for sufficiently small perturbation if the targets $\mathcal{A}_{i}^{V}$ are sufficiently small. In practice, given $\mathcal{A}$ one can determine appropriate $\mathcal{A}_{i}^{V}$ by numerical simulations.

\subsection{Conversion to a robust stabilization problem}

The small gain theorem tells us that we can achieve ultimate boundedness of $\mathcal{A}$ by designing an ISpS-controller for each subsystem $\Sigma_{i}$, considering the influence of the other states $\boldsymbol{x}_{j}, j \neq i$, as perturbations. To this end, we extend the perturbation of $\Sigma_{i}$ to $\boldsymbol{v}_{i}=\left(x_{1}, \ldots, x_{i-1}, x_{i+1}, \ldots, x_{N}, d_{i}\right) \in \mathcal{V}_{i}=$ $\mathcal{X}_{1} \times \ldots \times \mathcal{X}_{i-1} \times \mathcal{X}_{i+1} \times \ldots \times \mathcal{X}_{N} \times \mathcal{D}_{i}$ and write the subsystem as

$$
\boldsymbol{x}_{i}(k+1)=\boldsymbol{f}_{i}\left(\boldsymbol{x}_{i}(k), \boldsymbol{u}_{i}(k), \boldsymbol{v}_{i}(k)\right) .
$$

In order to design an ISpS controller for (9), we follow the approach in Grüne \& Sigurani (2013), which in turn is based on ideas from Jiang \& Wang (2001). This approach uses that system (5) is ISpS if and only if it is practically robustly stable. Practical robust stability means that there exists $\boldsymbol{e}_{i}: \mathcal{X}_{i} \times \widetilde{\mathcal{V}}_{i} \rightarrow$ $\mathcal{V}_{i}$ and $\eta_{i} \in \mathcal{K}_{\infty}$ such that the system

$$
\boldsymbol{x}_{i}(k+1)=\boldsymbol{f}_{i}\left(\boldsymbol{x}_{i}(k), \boldsymbol{u}_{\mathcal{P}_{i}}\left(\boldsymbol{x}_{i}(k)\right), \boldsymbol{e}_{i}\left(\boldsymbol{x}_{i}(k), \tilde{\boldsymbol{v}}_{i}(k)\right)\right)
$$

with $\widetilde{\mathcal{V}}_{i}=\bar{B}_{1}(0)$ is uniformly practically asymptotically stable ${ }^{1}$, where $\boldsymbol{e}_{i}$ is such that for each $\boldsymbol{v}_{i} \in \mathcal{V}_{i}$ with $\left\|\boldsymbol{v}_{i}\right\| \leq \eta_{i}\left(\left\|\boldsymbol{x}_{i}\right\|\right)$ there exists $\tilde{\boldsymbol{v}}_{i} \in \widetilde{\mathcal{V}}_{i}$ with $\boldsymbol{e}_{i}\left(\boldsymbol{x}_{i}, \tilde{\boldsymbol{v}}_{i}\right)=\boldsymbol{v}_{i}$. The resulting ISpS gains are discussed after Theorem 2, below.

\subsection{Solution of the robust stabilization problem}

In order to construct a controller rendering (9) uniformly practically asymptotically stable we employ the dynamic game approach from Grüne \& Junge (2007) which in turn relies on ideas from Grüne \& Junge (2008), Junge \& Osinga (2004). This approach introduces a quantization of the state space $\mathcal{X}_{i}$ using a finite partition $\mathcal{P}_{i}$ of boxes $P$.

Let a target set $\mathcal{A}_{i}^{V}$ be given which contains a neighborhood of the origin. We select a stage cost $g_{i}: \mathcal{X}_{i} \times \mathcal{U}_{i} \rightarrow \mathbb{R}$ which penalizes the distance to the origin and define the accumulated cost as

$$
J_{i}\left(\boldsymbol{x}_{i}^{0}, \underline{\boldsymbol{u}_{i}}, \underline{\tilde{\boldsymbol{v}}_{i}}\right):=\sum_{k=0}^{k_{i}\left(\mathcal{F}_{i}^{V}, \boldsymbol{x}_{i}^{0}, \underline{\boldsymbol{u}_{i}}, \underline{\tilde{\boldsymbol{v}}_{i}}\right)} g_{i}\left(\boldsymbol{x}_{i}\left(k, \boldsymbol{x}_{i}^{0}, \underline{\boldsymbol{u}_{i}}, \underline{\tilde{\boldsymbol{v}}_{i}}\right), \boldsymbol{u}_{i}(k)\right),
$$

\footnotetext{
${ }^{1}$ Practical asymptotic stability is defined like ISpS in Definition 2 but with $\gamma \equiv 0$.
}

where $k_{i}\left(\mathcal{A}_{i}^{V}, \boldsymbol{x}_{i}^{0}, \underline{\boldsymbol{u}_{i}}, \underline{\tilde{\boldsymbol{v}}_{i}}\right)$ denotes the smallest $k$ for which $\boldsymbol{x}_{i}\left(k, \boldsymbol{x}_{i}^{0}, \underline{\boldsymbol{u}_{i}}, \underline{\tilde{\boldsymbol{v}}_{i}}\right) \in \overline{\mathcal{A}_{i}^{V}} \overline{\text { holds. }}$.

The computation of $V_{i}$ is performed by a graph theoretic approximation of the dynamics of the system on the partition $\mathcal{P}_{i}$. Since the model includes both control and perturbation the resulting graph takes the form of a hypergraph. The computation of $V_{i}$ can then be carried out by solving a generalized min-max shortest path problem on this hypergraph. This yields an approximation $V_{\mathcal{P}_{i}}$ of $V_{i}$ which is constant on each element $P$ of the partition $\mathcal{P}_{i}$. An approximation $\boldsymbol{u}_{\mathcal{P}_{i}}$ of the optimal controller is obtained through the quantized dynamic programming principle

$\boldsymbol{u}_{\mathcal{P}_{i}}\left(\left[\boldsymbol{x}_{i}\right]\right):=\underset{\boldsymbol{u}_{i} \in U_{i}}{\operatorname{argmin}}\left\{\sup g_{i}\left(\left[\boldsymbol{x}_{i}\right], \boldsymbol{u}_{i}\right)+\sup _{v_{i} \in \widetilde{\mathcal{V}}_{i}} \sup _{\boldsymbol{x}_{i}^{\prime} \in \boldsymbol{f}_{i}\left(\left[\boldsymbol{x}_{i}\right], \boldsymbol{u}_{i}, v_{i}\right)} V_{\mathcal{P}_{i}}\left(\boldsymbol{x}_{i}^{\prime}\right)\right\}$.

This controller is defined on the stabilizable set w.r.t. $V_{\mathcal{P}_{i}}$ given by $S_{\mathcal{P}_{i}}:=\left\{\boldsymbol{x}_{i} \in \mathcal{X}_{i} \mid V_{\mathcal{P}_{i}}\left(\boldsymbol{x}_{i}\right)<\infty\right\}$. For details we refer to Grüne \& Junge (2007).

The following theorem in (Grüne \& Sigurani, 2013, Theorem 12) summarizes the properties of the controller $\boldsymbol{u}_{\mathcal{P}_{i}}$ derived this way.

Theorem 2. Consider the subsystem (9) satisfying the two inequalities before Theorem 1 and let $V_{\mathcal{P}_{i}}$ denote the approximate optimal value function constructed according to the algorithm presented in Grüne $\mathcal{F}$ Junge (2007) on a given partition $\mathcal{P}_{i}$ and target $\mathcal{A}_{i}^{V}$ for system (10). Denote the corresponding feedback by $\boldsymbol{u}_{\mathcal{P}_{i}}$. Let $\ell_{i} \leq \max _{\boldsymbol{s} \in S_{\mathcal{P}_{i}}} V_{\mathcal{P}_{i}}(s)$ and let $\underline{\alpha}_{i}, \bar{\alpha}_{i} \in \mathcal{K}_{\infty}$ denote functions such that (7) holds on $\mathcal{Y}_{i}=\left\{\boldsymbol{x}_{i} \in \bar{X}_{i} \mid V_{\mathcal{P}_{i}}\left(\boldsymbol{x}_{i}\right) \leq \ell_{i}\right\}$. Then, system (9) with controller $\boldsymbol{u}_{\mathcal{P}_{i}}$ is ISpS on $\mathcal{Y}_{i}$, with $\Delta_{d} \rightarrow \infty$ as $\ell_{i} \rightarrow \infty$ and $\delta_{i} \rightarrow 0$ as $\sup _{x_{i} \in \mathcal{F}_{i}^{V}}\left\|x_{i}\right\| \rightarrow 0$, provided $\delta_{i} \leq \underline{\alpha}_{i}^{-1}\left(\ell_{i}\right)$ holds.

The decentralized controller $\boldsymbol{u}_{\mathcal{P}}$ is obtained by carrying out the procedure just explained for all subsystems and defining $\boldsymbol{u}_{\mathcal{P}}(\boldsymbol{x}):=\left(\boldsymbol{u}_{\mathcal{P}_{1}}\left(\boldsymbol{x}_{1}\right), \ldots, \boldsymbol{u}_{\mathcal{P}_{N}}\left(\boldsymbol{x}_{N}\right)\right)$. The following remark summarizes two practical issues for setting up the global controller in our application.

Remark 1. a) The resulting Lyapunov gains can be estimated by the $\eta_{i}$ bounding the term $\boldsymbol{e}_{i}$ in (10) and the bounds on the $V_{i}$. More precisely, one can estimate $\mu_{i j} \leq$ $\bar{\alpha}_{i} \circ \eta_{i}^{-1} \circ \underline{\alpha}_{j}^{-1}$ and $\mu_{i} \leq \bar{\alpha}_{i} \circ \eta_{i}^{-1}$. Refined estimates are possible but we refrain from going into details here. In our application we have used numerical simulations in order to determine $\boldsymbol{e}_{i}$ which yield a stabilizing decentralized controller $\boldsymbol{u}_{\boldsymbol{P}}$.

b) The set $\mathcal{Y}$ on which $\boldsymbol{u}_{\mathcal{P}}$ is defined is given by the cartesian product $\mathcal{Y}=\mathcal{Y}_{1} \times \ldots \times \mathcal{Y}_{N}$ for the level sets $\mathcal{Y}_{i}$ from Theorem 2. Hence, in general $\mathcal{Y}$ may be smaller than $\mathcal{X}$. For the application considered in this paper, however, we were able to find Lyapunov functions $V_{i}$ with $S_{\mathcal{P}_{i}}=\mathcal{X}_{i}$ for all $i$ implying $\mathcal{Y}=\mathcal{X}$. 


\subsection{Operating principle of the global controller}

Above, we have described the discrete time version of the global approach. Its event-based implementation, as introduced and discussed in Grüne \& Müller (2009) and Grüne et al. (2010), is explained in this section.

The global controller for subsystem $\Sigma_{i}$ is obtained as a solution to the optimization problem (11) and is formulated as a look-up table which maps each element $P$ of the partition $\mathcal{P}_{i}$ to the control input $\boldsymbol{u}_{P}$. Within $P$ the control input $\boldsymbol{u}_{P}$ is kept constant and it only changes when the state moves from one partition element $P$ to another. Hence, an event is triggered whenever the state leaves a partition element, i.e, whenever the value of the quantization $\left[\boldsymbol{x}_{i}\right]$ changes.

Referring to the structure of the event-based controller as illustrated in Fig. 1, the global event-based controller for $\Sigma_{i}$ is subdivided into two components:

- The event generator $E_{i}$ continuously measures the quantized state $\left[x_{i}(t)\right]$ and detects the event time $t_{k_{i}}$ at which the state enters a new partition $P$. At this time $t_{k_{i}}$, the event generator $E_{i}$ transmits the information about the current partition $P$ to the control input generator $C_{i}$.

- The control input generator $C_{i}$ includes the look-up table. At the time $t_{k_{i}}$ it uses the received information about the partition $P=\left[\boldsymbol{x}_{i}(t)\right]$ to determine the control input $\boldsymbol{u}_{P}$. This control value is applied until the next event occurs.

Remark 2. Motivated by the successful practical application in this paper, the theoretical foundations of the event-based implementation of the proposed controller are currently under investigation. Note that existing approaches (De Persis et al. (2011)) use the small gain condition in order to generate events while in our case the task is to analyze an event based ISpS controller using small gain techniques.

\section{The local approach}

This section presents a decentralized event-based statefeedback approach which, once the state $\boldsymbol{x}_{i}(t)$ of subsystem $\Sigma_{i}$ has entered the target set $\mathcal{A}_{i}$ at time $T_{i}$, ensures that $\boldsymbol{x}_{i}(t) \in \mathcal{A}_{i}$ holds for $t \geq T_{i}$ in spite of disturbances $\boldsymbol{d}_{i}(t)$ and of the interconnections to the remaining subsystems. For the overall system, the relation $\boldsymbol{x}(t) \in \mathcal{A} \Leftrightarrow \boldsymbol{x}_{i}(t) \in \mathcal{A}_{i}$, for all $i \in \mathcal{N}$ holds (cf. Fig. 3).

\subsection{Models}

The target set $\mathcal{A}_{i}$ is a surrounding of the operating point $\overline{\boldsymbol{x}}_{i}$ of $\Sigma_{i}$, in which $\Sigma_{i}$ is described for $t \geq T_{i}$ by the linear state-space model

$$
\Sigma_{i}:\left\{\begin{aligned}
\dot{x}_{i}(t) & =\boldsymbol{A}_{i} \boldsymbol{x}_{i}(t)+\boldsymbol{B}_{i} \boldsymbol{u}_{i}(t)+\boldsymbol{E}_{i} \boldsymbol{d}_{i}(t)+\boldsymbol{E}_{\mathrm{si}} \boldsymbol{s}_{i}(t) \\
\boldsymbol{x}_{i}\left(T_{i}\right) & =\boldsymbol{x}_{\mathrm{T} i} \\
\boldsymbol{z}_{i}(t) & =\boldsymbol{C}_{\mathrm{zi}} \boldsymbol{x}_{i}(t),
\end{aligned}\right.
$$

where $\boldsymbol{s}_{i} \in \mathbb{R}^{p_{i}}$ and $\boldsymbol{z}_{i} \in \mathbb{R}^{q_{i}}$ denote the coupling input and coupling output, respectively. $\Sigma_{i}$ is interconnected with the remaining subsystems according to the relation

$$
\boldsymbol{s}_{i}(t)=\sum_{j=1}^{N} \boldsymbol{L}_{i j} \boldsymbol{z}_{j}(t),
$$

where $\boldsymbol{L}_{i i}=\mathbf{0}$ holds for all $i \in \mathcal{N}$ by assumption. The model (12), (13) is obtained from the nonlinear model (1) by means of linearization around the operating point $\overline{\boldsymbol{x}}_{i}$ for each $i \in \mathcal{N}$.

The control input generator $C_{i}$ and the event generator $E_{i}$ for $\Sigma_{i}$ are designed by using the method of Lunze \& Lehmann (2010), which is applied here for the isolated subsystems

$$
\dot{\boldsymbol{x}}_{i}(t)=\boldsymbol{A}_{i} \boldsymbol{x}_{i}(t)+\boldsymbol{B}_{i} \boldsymbol{u}_{i}(t)+\boldsymbol{E}_{i} \boldsymbol{d}_{i}(t), \quad \boldsymbol{x}_{i}\left(T_{i}\right)=\boldsymbol{x}_{\mathrm{T} i}, \quad i \in \mathcal{N}
$$

and leads to the components explained in the next sections.

\subsection{Components of the event-based control loops}

Control input generators $C_{i}$. In the interval $t \in\left[t_{k_{i}}, t_{k_{i}+1}\right)$, the control input generators $C_{i}$ are represented by the model

$$
C_{i}:\left\{\begin{array}{c}
\Sigma_{\mathrm{s} i}:\left\{\begin{array}{c}
\dot{\boldsymbol{x}}_{\mathrm{s} i}(t)=\overline{\boldsymbol{A}}_{i} \boldsymbol{x}_{\mathrm{s} i}(t)+\boldsymbol{E}_{i} \hat{\boldsymbol{d}}_{i}\left(t_{k_{i}}\right) \\
\boldsymbol{x}_{\mathrm{s} i}\left(t_{k_{i}}^{+}\right)=\boldsymbol{x}_{i}\left(t_{k_{i}}\right) \\
\boldsymbol{u}_{i}(t)=-\boldsymbol{K}_{i} \boldsymbol{x}_{\mathrm{s} i}(t)
\end{array}\right.
\end{array}\right.
$$

for $i \in \mathcal{N}$, where $\boldsymbol{x}_{\mathrm{s} i} \in \mathbb{R}^{n_{i}}$ denotes the state and $\hat{\boldsymbol{d}}_{i}\left(t_{k_{i}}\right)$ is a disturbance estimate. This event-based control approach works with any disturbance estimation method that yields bounded estimates $\hat{\boldsymbol{d}}_{i}\left(t_{k_{i}}\right)$, e.g. with the trivial estimation $\hat{\boldsymbol{d}}_{i}\left(t_{k_{i}}\right) \equiv \mathbf{0}$ for all $k_{i} \in \mathbb{N}_{0}$ or with the more sophisticated disturbance estimate presented in Stöcker et al. (2013). In (15), the state-feedback gain $\boldsymbol{K}_{i}$ is chosen such that the matrix

$$
\overline{\boldsymbol{A}}_{i}:=\boldsymbol{A}_{i}-\boldsymbol{B}_{i} \boldsymbol{K}_{i}
$$

is Hurwitz.

Event generators $E_{i}$. Like the control input generators, the event generators include the model $\Sigma_{\mathrm{s} i}$ defined in (15). In order to determine the event times $t_{k_{i}}\left(k_{i}=1,2, \ldots\right), E_{i}$ monitors the difference state

$$
\boldsymbol{x}_{\Delta i}(t):=\boldsymbol{x}_{i}(t)-\boldsymbol{x}_{\mathrm{si}}(t)
$$

and triggers an event, whenever the condition

$$
\left|\boldsymbol{x}_{\Delta i}(t)\right|=\overline{\boldsymbol{e}}_{i}
$$

is satisfied, where $\overline{\boldsymbol{e}}_{i} \in \mathbb{R}_{+}^{n_{i}}$ denotes the event threshold vector. The condition (16) is to be understood to hold element-wise, i.e., an event is triggered whenever one component of $\left|\boldsymbol{x}_{\Delta i}(t)\right|$ equals the corresponding component of the vector $\overline{\boldsymbol{e}}_{i}$. Hence, $E_{i}$ continuously measures the subsystem state $\boldsymbol{x}_{i}(t)$ and it determines the event times $t_{k_{i}}$ using the model

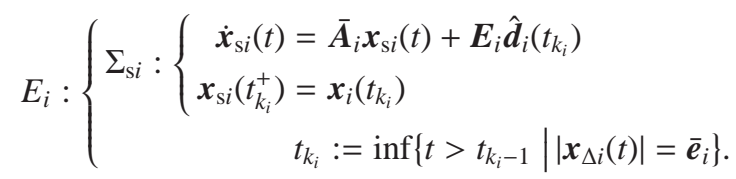


At the event time $t_{k_{i}}, E_{i}$ transmits the current subsystem state $\boldsymbol{x}_{i}\left(t_{k_{i}}\right)$ to $C_{i}$ and this information is used in both components to reset the state $\boldsymbol{x}_{\mathrm{s} i}$ of the model $\Sigma_{\mathrm{s} i}$, which implies $\boldsymbol{x}_{\Delta i}\left(t_{k_{i}}^{+}\right)=\mathbf{0}$ for all event times $t_{k_{i}}, k_{i} \in \mathbb{N}_{0}$.

\subsection{Stability analysis}

The control input generator $C_{i}$ and the event generator $E_{i}$ are designed under the assumption of vanishing interconnections $\left(s_{i}(t)=\mathbf{0}\right.$ for all $\left.i \in \mathcal{N}\right)$ and with the aim to ensure the stability of the isolated event-based control loops. This section presents a condition on the interconnection matrix $\boldsymbol{L}$ for which the stability of the isolated event-based control loops implies the stability of the overall control system.

The following theorem summarizes a stability test which has been derived by Stöcker et al. (2013) using the comparison principle (see Lunze (1992)).

Theorem 3 (Stöcker et al. (2013)). The overall event-based control system that consists of the interconnected subsystems (12), (13) and the decentralized event-based controllers (15),

(17) is ultimately bounded if the condition

$$
\lambda_{\mathrm{P}}\left(\int_{0}^{\infty} \overline{\boldsymbol{G}}_{\mathrm{xS}}(t) \overline{\boldsymbol{L}} \overline{\boldsymbol{C}}_{\mathrm{z}} \mathrm{d} t\right)<1
$$

is satisfied with

$$
\begin{aligned}
\overline{\boldsymbol{G}}_{\mathrm{xs}}(t) & =\operatorname{diag}\left(\left|\mathrm{e}^{\overline{\boldsymbol{A}}_{i} t} \boldsymbol{E}_{\mathrm{s} i}\right|\right), \\
\overline{\boldsymbol{C}}_{\mathrm{z}} & =\operatorname{diag}\left(\left|\boldsymbol{C}_{\mathrm{zi}}\right|\right)
\end{aligned}
$$

The matrix

$$
\overline{\boldsymbol{L}}=\left(\begin{array}{cccc}
\mathbf{0} & \left|\boldsymbol{L}_{12}\right| & \ldots & \left|\boldsymbol{L}_{1 N}\right| \\
\left|\boldsymbol{L}_{21}\right| & \mathbf{0} & \ldots & \left|\boldsymbol{L}_{2 N}\right| \\
\vdots & \vdots & \ddots & \vdots \\
\left|\boldsymbol{L}_{N 1}\right| & \left|\boldsymbol{L}_{N 2}\right| & \ldots & \mathbf{0}
\end{array}\right)
$$

represents a bound on the interconnections among the subsystems.

The stability condition (18) is a small-gain condition requiring the interconnection among the subsystems to be sufficiently weak. Hence, (18) can be used to find a bound on the interconnection up to which the stability of the overall system is guaranteed.

The following result explicitly characterizes a region $\mathcal{B}$ in which the state $\boldsymbol{x}(t)$ of the overall system is maintained by the decentralized event-based controller (15), (17).

Theorem 4 (Stöcker et al. (2013)). Consider the interconnected subsystems (12), (13) together with the decentralized event-based controllers (15), (17) and assume that the condition (18) is satisfied. The set

$$
\mathcal{B}:=\left\{\boldsymbol{x}=\left(\boldsymbol{x}_{1}^{\top}, \ldots, \boldsymbol{x}_{N}^{\top}\right)^{\top} \in \mathbb{R}^{n} \mid\left(\left|\boldsymbol{x}_{1}\right|, \ldots,\left|\boldsymbol{x}_{N}\right|\right)^{\top} \leq \boldsymbol{b}(\boldsymbol{\varepsilon}, \boldsymbol{\delta})\right\}
$$

is positive invariant for the overall control system (12), (13), (15), (17), with the ultimate bound

$$
\boldsymbol{b}(\boldsymbol{\varepsilon}, \boldsymbol{\delta})=\left(\boldsymbol{I}_{n}-\int_{0}^{\infty} \overline{\boldsymbol{G}}_{\mathrm{xs}}(t) \overline{\boldsymbol{L}} \overline{\boldsymbol{C}}_{\mathrm{z}} \mathrm{d} t\right)^{-1}(\boldsymbol{\varepsilon}+\boldsymbol{\delta})
$$

where

$$
\begin{aligned}
& \boldsymbol{\varepsilon}=\int_{0}^{\infty} \operatorname{diag}\left(\left|\mathrm{e}^{\overline{\boldsymbol{A}}_{i} t} \boldsymbol{B}_{i} \boldsymbol{K}_{i}\right|\right) \mathrm{d} t \cdot\left(\overline{\boldsymbol{e}}_{1}^{\top}, \ldots, \overline{\boldsymbol{e}}_{N}^{\top}\right)^{\top}, \\
& \boldsymbol{\delta}=\int_{0}^{\infty} \operatorname{diag}\left(\left|\mathrm{e}^{\overline{\boldsymbol{A}}_{i} t} \boldsymbol{E}_{i}\right|\right) \mathrm{d} t \cdot\left(\overline{\boldsymbol{d}}_{1}^{\top}, \ldots, \overline{\boldsymbol{d}}_{N}^{\top}\right)^{\top}
\end{aligned}
$$

and the matrices $\overline{\boldsymbol{G}}_{\mathrm{xS}}(t), \overline{\boldsymbol{C}}_{\mathrm{z}}$ and $\overline{\boldsymbol{L}}$ are given in (19)-(21).

The inverse matrix in (23) exists if the condition (18) is satisfied. Theorem 4 shows that the size of the set $\mathcal{B}$ depends upon the disturbance magnitudes $\overline{\boldsymbol{d}}_{i}$ and the event thresholds $\overline{\boldsymbol{e}}_{i}$ and that it can be adjusted by appropriately setting the event thresholds $\overline{\boldsymbol{e}}_{i}$ for all $i \in \mathcal{N}$.

\section{Combination of the global and the local approach}

This section explains how the global and the local approach are merged in order to obtain an event-based controller that renders the system ultimately bounded. The overall event-based controller combines the local and the global approaches by including in each component the functionalities of both.

Besides the previously defined triggering conditions, all event generators $E_{i}$ also includes the logic that induces the switching from the global to the local approach in both components. The switching time for the event-based controller for subsystem $\Sigma_{i}$ is given by

$$
T_{i}:=\inf \left\{t \geq 0 \mid \boldsymbol{x}_{i}(t) \in \mathcal{A}_{i}\right\}
$$

At this time, $E_{i}$ switches the functionality from the global to the local approach and transmits a respective command to the corresponding control input generator $C_{i}$. The local approach keeps the state $\boldsymbol{x}_{i}(t)$ within the target set $\mathcal{A}_{i}$ for all $t \geq T_{i}$. Note that the decentralized event-based controllers decide locally at which time they switch from the global to the local approach and, thus, the switching occurs asynchronously in time (Fig. 3).

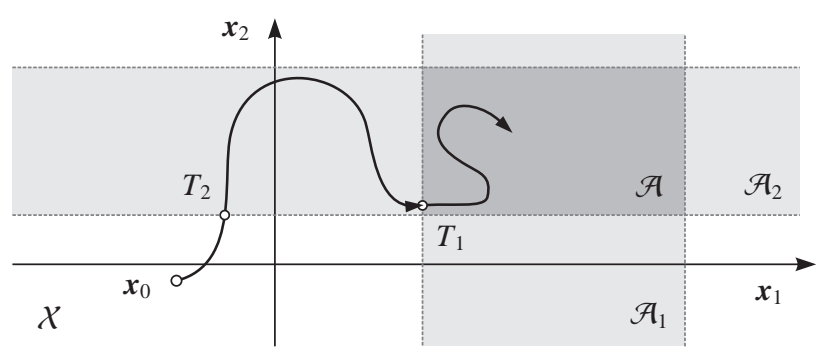

Figure 3: Switching from the global to the local approach 


\section{Application to a continuous flow process}

\subsection{Hardware description}

The proposed event-based control is tested and evaluated on the pilot plant at the Institute of Automation and Computer Control at Ruhr-University Bochum, Germany (Fig. 4). The plant includes four cylindrical storage tanks, three batch reactors and a buffer tank which are connected over a complex pipe system and it is constructed with standard industrial components including more than 70 sensors and 80 actuators.

Figure 5 illustrates the automation concept for the pilot plant which is subdivided into three layers. On the top layer, the event-based control is implemented on an ordinary personal computer (PC). The functionalities of the control input generators $C_{i}$ and event generators $E_{i}$ are realized in MATLAB/Simulink executed with the sampling time $T_{\mathrm{s}}=0.2 \mathrm{~s}$. The PC is connected over a $100 \mathrm{Mbit} / \mathrm{s}$ Ethernet network with the programmable logic controllers (PLCs) on which subordinate controllers and several routines for the plant protection are implemented. On the field level (Sensor/actuator control) the actuator signals are applied and the sensor signals are sampled via the perepherials A to E that are connected over PROFIBUS DP with the PLCs.

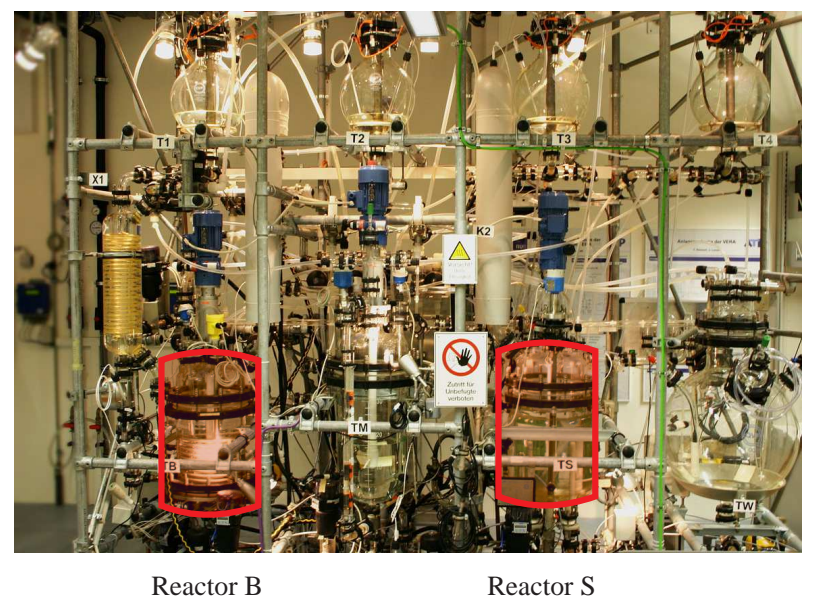

Figure 4: Pilot plant. The reactors which are used for the considered process are highlighted.

\subsection{Process description}

The experimental setup is illustrated in Fig. 6. The main components are the two reactors $\mathrm{B}$ and $\mathrm{S}$ in which continuous flow processes shall be realized. Reactor B is connected to the storage tank $\mathrm{T}_{1}$ from where the inflow can be controlled by means of the valve angle $u_{\mathrm{T} 1}$. Via the pump PB a part of the outflow is pumped out into the buffer tank TW (and is not used further in the process) while the remaining outflow is conducted to the reactor $\mathrm{S}$. The temperature $\vartheta_{\mathrm{B}}(t)$ of the water in reactor $\mathrm{B}$ is influenced by the cooling unit (CU) using the input $u_{\mathrm{CU}}$ or by the heating rods that are driven by the signal $d_{\mathrm{H}}$. The inflow from the storage tank $T_{3}$ to the reactor $S$ can be adjusted by means of the opening angle $u_{\mathrm{T} 3}$. Reactor $\mathrm{S}$ is additionally fed by the fresh water supply (FW) from where the inflow is set by

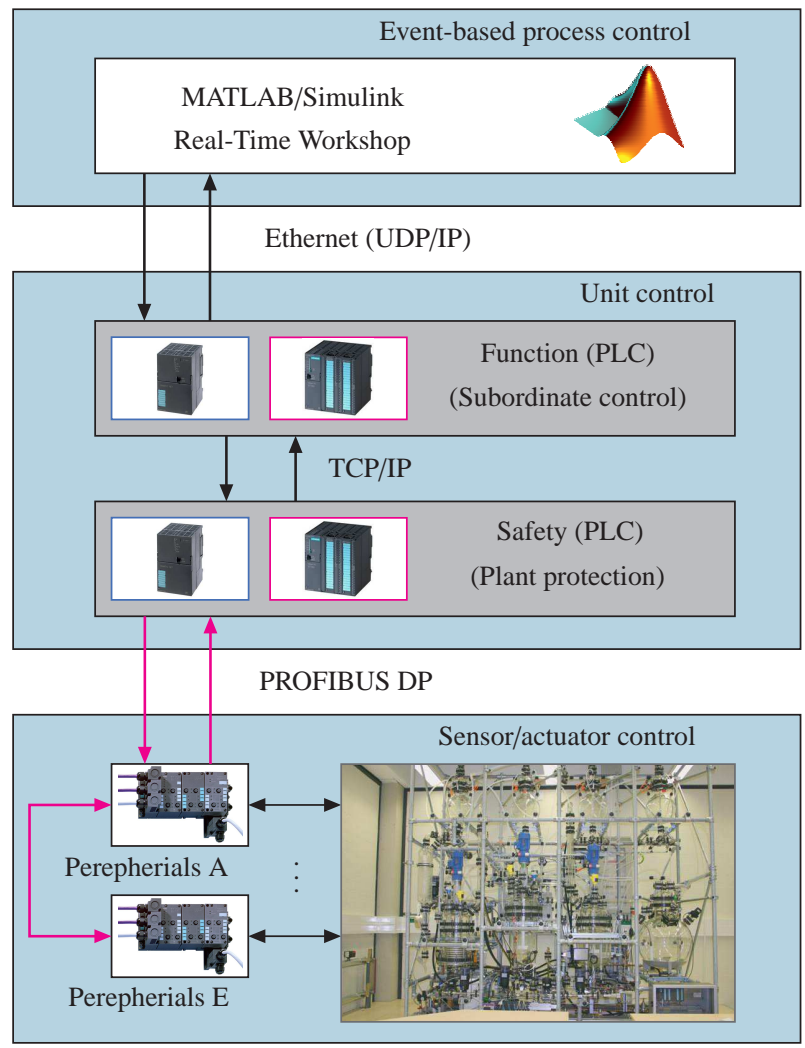

Figure 5: Automation concept for the pilot plant

means of the valve angle $d_{\mathrm{F}}$. Equivalently to reactor B, the outflow of reactor $\mathrm{S}$ is split and one part is conveyed via the pump PS to TW and the other part is pumped to the reactor B. The temperature $\vartheta_{\text {TS }}(t)$ of the liquid in reactor $\mathrm{S}$ can be increased by the heating rods that are controlled by the signal $u_{\mathrm{H}}$.

The two reactors are coupled by the flow from reactor B to reactor $\mathrm{S}$ and vice versa, where the coupling strength can be adjusted by means of the valve angles $u_{\mathrm{BS}}$ and $u_{\mathrm{SB}}$. The ratio of the volume that is used for the coupling of the systems and the outflow to TW is set by the valve angles $u_{\mathrm{BW}}$ and $u_{\mathrm{SW}}$. Both reactor $B$ and reactor $S$ are equipped with sensors that continuously measure the level and the temperature of the contents.

\subsection{Plant model}

In the following, the behavior of the level and the temperature in reactor $\mathrm{B}$ and reactor $\mathrm{S}$ are considered as subsystem $\Sigma_{1}$ and $\Sigma_{2}$, respectively. Hence, the states of the subsystems are represented by $\boldsymbol{x}_{1}(t)=\left(l_{\mathrm{B}}(t) \vartheta_{\mathrm{B}}(t)\right)^{\top}$ and $\boldsymbol{x}_{2}(t)=\left(l_{\mathrm{S}}(t) \vartheta_{\mathrm{S}}(t)\right)^{\top}$. The continuous flow process is represented by the nonlinear state- 


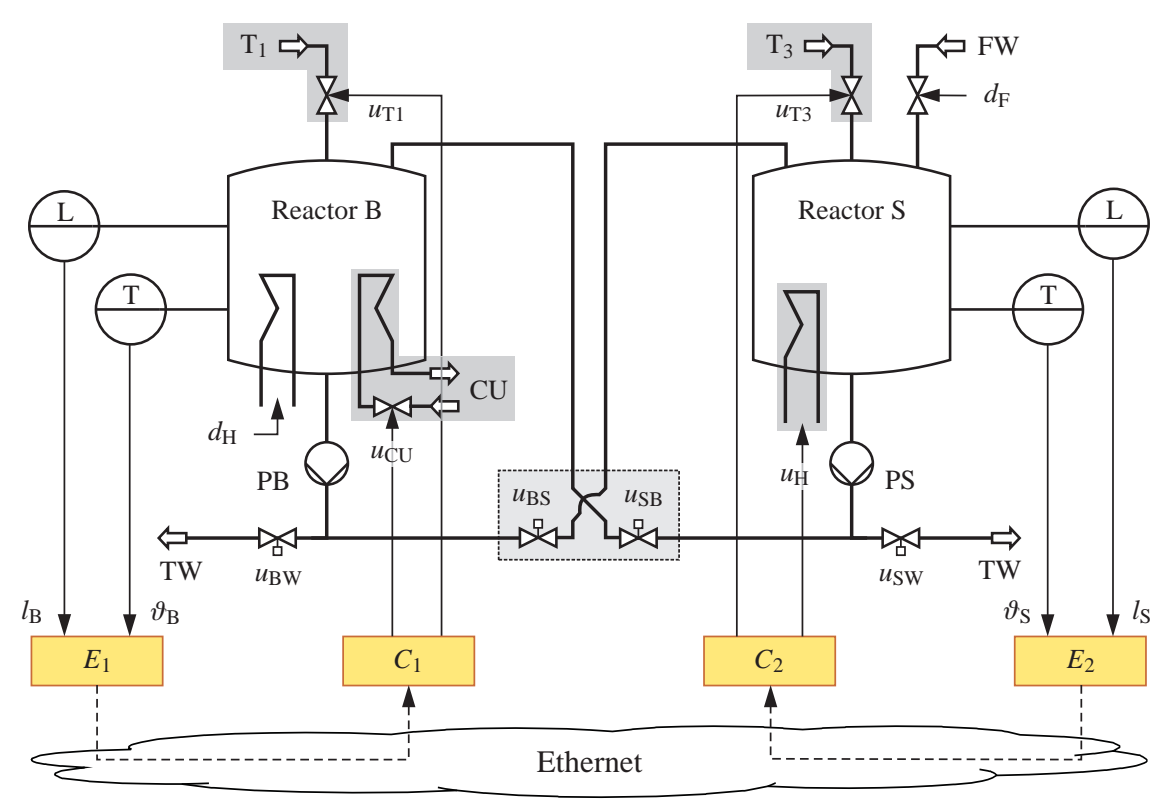

Figure 6: Experimental setup of the continuous flow process

space model

$$
\begin{aligned}
& \dot{l}_{\mathrm{B}}(t)=A_{\mathrm{B}}^{-1}\left(q_{1 \mathrm{~B}}\left(u_{\mathrm{T} 1}(t)\right)+q_{\mathrm{SB}}\left(l_{\mathrm{S}}(t), u_{\mathrm{SB}}\right)-q_{\mathrm{BW}}\left(l_{\mathrm{B}}(t), u_{\mathrm{BW}}\right)\right. \\
& \left.-q_{\mathrm{BS}}\left(l_{\mathrm{B}}(t), u_{\mathrm{BS}}\right)\right) \\
& \dot{\vartheta}_{\mathrm{B}}(t)=\left(A_{\mathrm{B}} l_{\mathrm{B}}(t)\right)^{-1}\left(q_{1 \mathrm{~B}}\left(u_{\mathrm{T} 1}(t)\right)\left(\vartheta_{1}-\vartheta_{\mathrm{B}}(t)\right)\right. \\
& +q_{\mathrm{SB}}\left(l_{\mathrm{S}}(t), u_{\mathrm{SB}}\right)\left(\vartheta_{\mathrm{S}}(t)-\vartheta_{\mathrm{B}}(t)\right) \\
& \left.+q_{\mathrm{C}}\left(u_{\mathrm{CU}}(t)\right)\left(\vartheta_{\mathrm{C}}-\vartheta_{\mathrm{B}}(t)\right)+H_{\mathrm{B}} d_{\mathrm{H}}(t)\right) \\
& \dot{l}_{\mathrm{S}}(t)=A_{\mathrm{S}}^{-1}\left(q_{3 \mathrm{~S}}\left(u_{\mathrm{T} 3}(t)\right)+q_{\mathrm{BS}}\left(l_{\mathrm{B}}(t), u_{\mathrm{BS}}\right)-q_{\mathrm{SW}}\left(l_{\mathrm{S}}(t), u_{\mathrm{SW}}\right)\right. \\
& \left.-q_{\mathrm{SB}}\left(l_{\mathrm{S}}(t), u_{\mathrm{SB}}\right)+q_{\mathrm{FS}}\left(d_{\mathrm{F}}(t)\right)\right) \\
& \dot{\vartheta}_{\mathrm{S}}(t)=\left(A_{\mathrm{S}} l_{\mathrm{S}}(t)\right)^{-1}\left(q_{3 \mathrm{~S}}\left(u_{\mathrm{T} 3}(t)\right)\left(\vartheta_{3}-\vartheta_{\mathrm{S}}(t)\right)\right. \\
& +q_{\mathrm{BS}}\left(l_{\mathrm{B}}(t), u_{\mathrm{BS}}\right)\left(\vartheta_{\mathrm{B}}(t)-\vartheta_{\mathrm{S}}(t)\right) \\
& \left.+q_{\mathrm{FS}}\left(d_{\mathrm{F}}(t)\right)\left(\vartheta_{\mathrm{F}}-\vartheta_{\mathrm{S}}(t)\right)+H_{\mathrm{S}} u_{\mathrm{H}}(t)\right)
\end{aligned}
$$

Here,

$$
\begin{aligned}
& q_{1 \mathrm{~B}}\left(u_{\mathrm{T} 1}(t)\right)=1.61 \times 10^{-4} \cdot u_{\mathrm{T} 1}(t) \\
& q_{3 \mathrm{~S}}\left(u_{\mathrm{T} 3}(t)\right)=1.81 \times 10^{-4} \cdot u_{\mathrm{T} 3}(t)
\end{aligned}
$$

denote the flows from the storage tanks $\mathrm{T}_{1}$ and $\mathrm{T}_{3}$ to the reactors $B$ and $S$, respectively.

$$
q_{\mathrm{C}}\left(u_{\mathrm{CU}}(t)\right)=0.97 \times 10^{-4} \cdot u_{\mathrm{CU}}(t)
$$

is the flow of the coolant and

$$
\begin{aligned}
q_{\mathrm{BS}}\left(l_{\mathrm{B}}(t), u_{\mathrm{BS}}\right) & =K_{\mathrm{BS}}\left(u_{\mathrm{BS}}\right) \sqrt{2 g l_{\mathrm{B}}(t)} \\
K_{\mathrm{BS}}\left(u_{\mathrm{BS}}\right) & =10^{-4} \cdot \begin{cases}1.02 \cdot u_{\mathrm{BS}}, & 0 \leq u_{\mathrm{BS}} \leq 0.1 \\
2.13 \cdot u_{\mathrm{BS}}-0.11, & 0.1<u_{\mathrm{BS}} \leq 1\end{cases} \\
q_{\mathrm{SB}}\left(l_{\mathrm{S}}(t), u_{\mathrm{SB}}\right) & =K_{\mathrm{SB}}\left(u_{\mathrm{SB}}\right) \sqrt{2 g l_{\mathrm{S}}(t)} \\
K_{\mathrm{SB}}\left(u_{\mathrm{SB}}\right) & =10^{-4} \cdot \begin{cases}0.90 \cdot u_{\mathrm{SB}}, & 0 \leq u_{\mathrm{SB}} \leq 0.1 \\
1.68 \cdot u_{\mathrm{SB}}-0.08, & 0.1<u_{\mathrm{SB}} \leq 1\end{cases}
\end{aligned}
$$

denote the flows from reactor $\mathrm{B}$ to reactor $\mathrm{S}$ and vice versa with the specific valve parameters $K_{\mathrm{BS}}$ and $K_{\mathrm{SB}}\left(\mathrm{m}^{3} / \mathrm{m}\right)$. Finally,

$$
\begin{aligned}
q_{\mathrm{BW}}\left(l_{\mathrm{B}}(t), u_{\mathrm{BW}}\right)=K_{\mathrm{BW}}\left(u_{\mathrm{TB}}\right) \sqrt{2 g l_{\mathrm{B}}(t)} & (27 \mathrm{f}) \\
K_{\mathrm{BW}}\left(u_{\mathrm{BW}}\right) & =10^{-4} \cdot\left\{\begin{array}{lr}
0.96 \cdot u_{\mathrm{TB}}, & 0 \leq u_{\mathrm{BW}} \leq 0.1 \\
2.01 \cdot u_{\mathrm{TB}}-0.10, & 0.1<u_{\mathrm{BW}} \leq 1
\end{array}\right. \\
q_{\mathrm{SW}}\left(l_{\mathrm{S}}(t), u_{\mathrm{SW}}\right) & =K_{\mathrm{SW}}\left(u_{\mathrm{SW}}\right) \sqrt{2 g l_{\mathrm{S}}(t)} \\
K_{\mathrm{SW}}\left(u_{\mathrm{SW}}\right) & =10^{-4} \cdot \begin{cases}0.79 \cdot u_{\mathrm{SW}}, & 0 \leq u_{\mathrm{SW}} \leq 0.1 \\
1.42 \cdot u_{\mathrm{SW}}-0.06, & 0.1<u_{\mathrm{SW}} \leq 1\end{cases}
\end{aligned}
$$

denote flows of volume from the reactors $B$ and $S$ into the buffer reactor TW with the specific valve parameters $K_{\mathrm{BW}}$ and $K_{\text {SW }}\left(\mathrm{m}^{3} / \mathrm{m}\right)$. All flows have the unit $\mathrm{m}^{3} / \mathrm{s}$. All parameters are listed in Table 1.

\begin{tabular}{lll}
\multicolumn{3}{c}{ Table 1: Parameters } \\
\hline Param. & Value & Meaning \\
\hline$A_{\mathrm{B}}$ & $0.07 \mathrm{~m}^{2}$ & Cross sectional area of tank B \\
$A_{\mathrm{S}}$ & $0.07 \mathrm{~m}^{2}$ & Cross sectional area of tank S \\
$g$ & $9.81 \mathrm{~m} / \mathrm{s}^{2}$ & Gravitation constant \\
$H_{\mathrm{B}}$ & $4.8 \times 10^{-3} \mathrm{~m}^{3} \mathrm{~K} / \mathrm{s}$ & Heat coefficient of heating in tank B \\
$H_{\mathrm{S}}$ & $0.8 \times 10^{-3} \mathrm{~m}^{3} \mathrm{~K} / \mathrm{s}$ & Heat coefficient of heating in tank S \\
$\vartheta_{1}$ & $294.15 \mathrm{~K}$ & Temperature of the fluid in tank $T_{1}$ \\
$\vartheta_{3}$ & $294.15 \mathrm{~K}$ & Temperature of the fluid in tank $T_{3}$ \\
$\vartheta_{\mathrm{C}}$ & $282.65 \mathrm{~K}$ & Temperature of the coolant \\
$\vartheta_{\mathrm{F}}$ & $294.15 \mathrm{~K}$ & Temperature of the water supply \\
\hline
\end{tabular}

Due to technical limitations the subsystem states $\boldsymbol{x}_{1}=$ $\left(l_{\mathrm{B}} \vartheta_{\mathrm{B}}\right)^{\top}$ and $x_{2}=\left(l_{\mathrm{S}} \vartheta_{\mathrm{S}}\right)^{\top}$ are restricted to the state space $\mathcal{X}=\mathcal{X}_{1} \times \mathcal{X}_{2}$ with

$$
\begin{aligned}
& X_{1}=[0.26 ; 0.40] \mathrm{m} \times[285.65 ; 323.15] \mathrm{K} \\
& \mathcal{X}_{2}=[0.26 ; 0.40] \mathrm{m} \times[293.15 ; 323.15] \mathrm{K} .
\end{aligned}
$$


The control inputs $\boldsymbol{u}_{1}=\left(u_{\mathrm{T} 1} u_{\mathrm{CU}}\right)^{\top}$ and $\boldsymbol{u}_{2}=\left(u_{\mathrm{T} 3} u_{\mathrm{H}}\right)^{\top}$ are limited to the set $\mathcal{U}=\mathcal{U}_{1} \times \mathcal{U}_{2}$ with

$$
\mathcal{U}_{1}=[0 ; 1] \times[0 ; 1], \quad \mathcal{U}_{2}=[0 ; 1] \times[0 ; 1]
$$

Note that the components which are used for the control are highlighted in gray in Fig. 6. The disturbance characteristics are accomplished by means of the heating with disturbance input $d_{1}(t)=d_{\mathrm{H}}(t)$ in reactor $\mathrm{B}$ and the additional water inflow in reactor $\mathrm{S}$ that is set by the valve angle $d_{2}(t)=d_{\mathrm{F}}(t)$. The disturbances are considered to be bounded to

$$
d_{1} \in \mathcal{D}_{1}=[0 ; 0.1], \quad d_{2} \in \mathcal{D}_{2}=[0 ; 0.25] .
$$

\subsection{Specification of the control aim}

The state $\boldsymbol{x}(t)$ of the overall system shall be steered from a given initial state $\boldsymbol{x}_{0} \in \mathcal{X}$ into the target region $\mathcal{A}=\mathcal{A}_{1} \times \mathcal{A}_{2}$ with

$$
\begin{aligned}
& \mathcal{A}_{1}=[0.3 ; 0.36] \mathrm{m} \times[291.7 ; 297.7] \mathrm{K} \\
& \mathcal{A}_{2}=[0.31 ; 0.37] \mathrm{m} \times[297.2 ; 303.2] \mathrm{K}
\end{aligned}
$$

around the operating point

$$
\overline{\boldsymbol{x}}_{1}=\left(\begin{array}{c}
\bar{l}_{\mathrm{B}} \\
\bar{\vartheta}_{\mathrm{B}}
\end{array}\right)=\left(\begin{array}{c}
0.33 \mathrm{~m} \\
294.7 \mathrm{~K}
\end{array}\right), \quad \overline{\boldsymbol{x}}_{2}=\left(\begin{array}{c}
\bar{l}_{\mathrm{S}} \\
\bar{\vartheta}_{\mathrm{S}}
\end{array}\right)=\left(\begin{array}{c}
0.34 \mathrm{~m} \\
300.2 \mathrm{~K}
\end{array}\right)
$$

and maintained in $\mathcal{A}$ for all time in spite of the influence of disturbances given in (30) and interconnections. The interconnections among both subsystems are set by the valve angles $u_{\mathrm{BS}}$ and $u_{\mathrm{SB}}$ which are fixed to

$$
u_{\mathrm{BS}}=0.19, \quad u_{\mathrm{SB}}=0.22
$$

throughout the experiments. Moreover, the choice

$$
u_{\mathrm{BW}}=0.21, \quad u_{\mathrm{SW}}=0.29
$$

defines the outflow from the reactors B and $\mathrm{S}$ to the buffer tank TW.

\subsection{Decentralized event-based controller resulting from the global approach}

The global approach calculates a decentralized event-based controller for each subsystem $\Sigma_{1}$ and $\Sigma_{2}$, utilizing the algorithm described in Section 3. To obtain the discrete time system (4) we use a sampling time of 2 seconds. For the numerical solution of the robust stabilization problem in Section 3.4 we approximate its solutions by a Runge-Kutta $(4,5)$ scheme with automatic step size control. For the quantization of each of the state spaces $\mathcal{X}_{1}$ and $\mathcal{X}_{2}$ from (28) we use a partition $\mathcal{P}$ of $8 \times 8$ equally sized rectangular elements. This relatively small number of regions turned out to be sufficient for computing value functions $V_{i}$ which are finite on $\mathcal{X}_{i}$. The target set $\mathcal{A}^{V}$ consists of the partition element containing the operating point (32), i.e., $\mathcal{A}_{1}^{V}=[0.33 ; 0.3475] \times[290.3375 ; 295.025]$ and $\mathcal{A}_{2}^{V}=[0.33 ; 0.3475] \times[296.9 ; 300.65]$, i.e., we choose a smaller set than $\mathcal{A}$ in (31). Our design is without external disturbances, i.e., we set $d_{\mathrm{H}}=d_{\mathrm{F}}=0$ and consider only the state of the other subsystem as disturbance by setting $\boldsymbol{v}_{1}=\left(l_{\mathrm{S}} \vartheta_{\mathrm{S}}\right)^{\top}$ and $\boldsymbol{v}_{2}=\left(l_{\mathrm{B}} \vartheta_{\mathrm{B}}\right)^{\top}$. The functions

$\boldsymbol{e}_{1}\left(\boldsymbol{x}_{1}, \tilde{\boldsymbol{v}}_{1}\right)=\left(\begin{array}{l}0.34+\sqrt{1.28\left(l_{\mathrm{B}}-0.33\right)^{2}} \tilde{\boldsymbol{v}}_{11} \\ 300.2+\sqrt{1053.4\left(l_{\mathrm{B}}-0.33\right)^{2}+0.63\left(\vartheta_{\mathrm{B}}-294.7\right)^{2}} \tilde{\boldsymbol{v}}_{12}\end{array}\right)$

and

$\boldsymbol{e}_{2}\left(\boldsymbol{x}_{2}, \tilde{\boldsymbol{v}}_{2}\right)=\left(\begin{array}{l}0.33+\sqrt{0.750312\left(l_{\mathrm{S}}-0.34\right)^{2}} \tilde{\boldsymbol{v}}_{21} \\ 294.7+\sqrt{1239.4\left(l_{\mathrm{S}}-0.34\right)^{2}+1.49\left(\vartheta_{\mathrm{S}}-300.2\right)^{2}} \tilde{\boldsymbol{v}}_{12}\end{array}\right)$

are used to convert the problem into a robust stabilization problem, thus obtaining system (10) with $\tilde{\boldsymbol{v}}_{1}=\left(\tilde{\boldsymbol{v}}_{11} \tilde{\boldsymbol{v}}_{12}\right)^{\top} \in[-1 ; 1]^{2}$ and $\tilde{\boldsymbol{v}}_{2}=\left(\tilde{\boldsymbol{v}}_{21} \tilde{\boldsymbol{v}}_{22}\right)^{\top} \in[-1 ; 1]^{2}$. Note that both subsystems have a cascaded (or triangular) structure. It has turned out beneficial to choose $\boldsymbol{e}_{i}$ to reflect this structure, i.e., the first components of the $\boldsymbol{e}_{i}$ are independent of $\vartheta_{\mathrm{B}}$ or $\vartheta_{\mathrm{S}}$, respectively.

For constructing the hypergraph we discretize the control input set for $\Sigma_{1}$ by $9 \times 5$ equidistant values, for $\Sigma_{2}$ by $9 \times 4$ equidistant values and the perturbation input set by choosing $\tilde{\boldsymbol{v}}_{i} \in\{-1 ; 0 ; 1\}^{2}$. A finer discretization did not yield significantly different results. The stage costs are chosen as

$$
g_{1}\left(\boldsymbol{x}_{1}, \boldsymbol{u}_{1}\right)=\frac{1}{0.0196}\left(l_{\mathrm{B}}-0.33\right)^{2}+\frac{1}{1406.25}\left(\vartheta_{\mathrm{B}}-294.7\right)^{2}
$$

and

$$
g_{2}\left(\boldsymbol{x}_{2}, \boldsymbol{u}_{2}\right)=\frac{1}{0.0196}\left(l_{\mathrm{S}}-0.34\right)^{2}+\frac{1}{1406.25}\left(\vartheta_{\mathrm{S}}-300.2\right)^{2}
$$

As mentioned in Section 3.4, an event is triggered whenever the state leaves a partition element.

The resulting approximated optimal value functions $V_{1}$ of $\Sigma_{1}$ (left) and $V_{2}$ of $\Sigma_{2}$ (right) depending on the initial value are depicted in Figure 6.5. One notes that the values of $V_{2}$ are much higher than those of $V_{1}$ because the cooling unit in reactor B is much slower than the heating unit in reactor $\mathrm{S}$. This effect is also visible when comparing the maximal time needed to reach the target set from a given partition element which is shown in Figure 6.5.

\subsection{Decentralized event-based controller resulting from the lo- cal approach}

As the local approach uses a linear model of the continuous flow process, the nonlinear system (26), (27) is linearized around the operating point (32) with

$$
\overline{\boldsymbol{u}}_{1}=\left(\begin{array}{c}
\bar{u}_{\mathrm{T} 1} \\
\bar{u}_{\mathrm{CU}}
\end{array}\right)=\left(\begin{array}{c}
0.5 \\
0.5
\end{array}\right), \quad \overline{\boldsymbol{u}}_{2}=\left(\begin{array}{c}
\bar{u}_{\mathrm{T} 3} \\
\bar{u}_{\mathrm{H}}
\end{array}\right)=\left(\begin{array}{c}
0.5 \\
0.5
\end{array}\right) .
$$



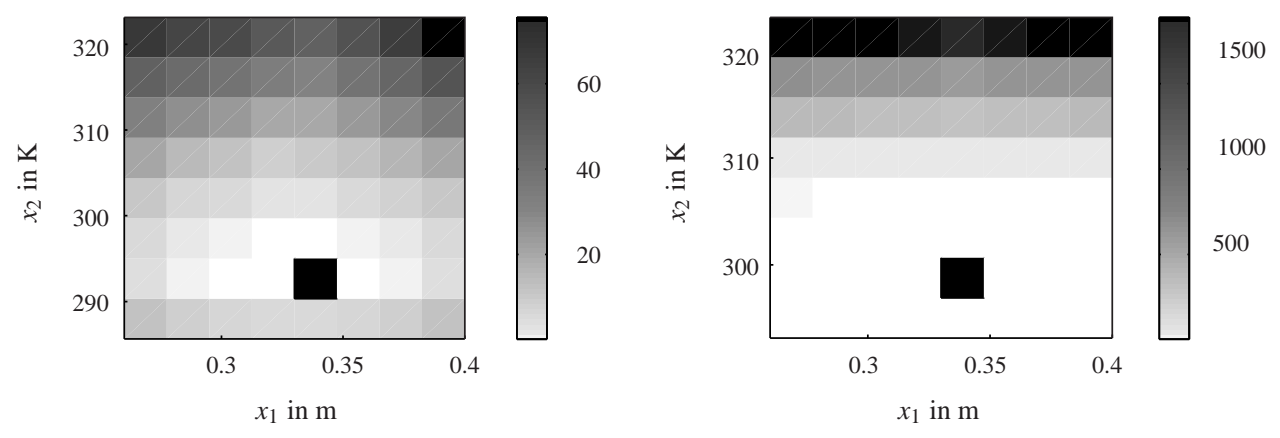

Figure 7: Value functions for $\Sigma_{1}$ (left) and $\Sigma_{2}$ (right) over state-space
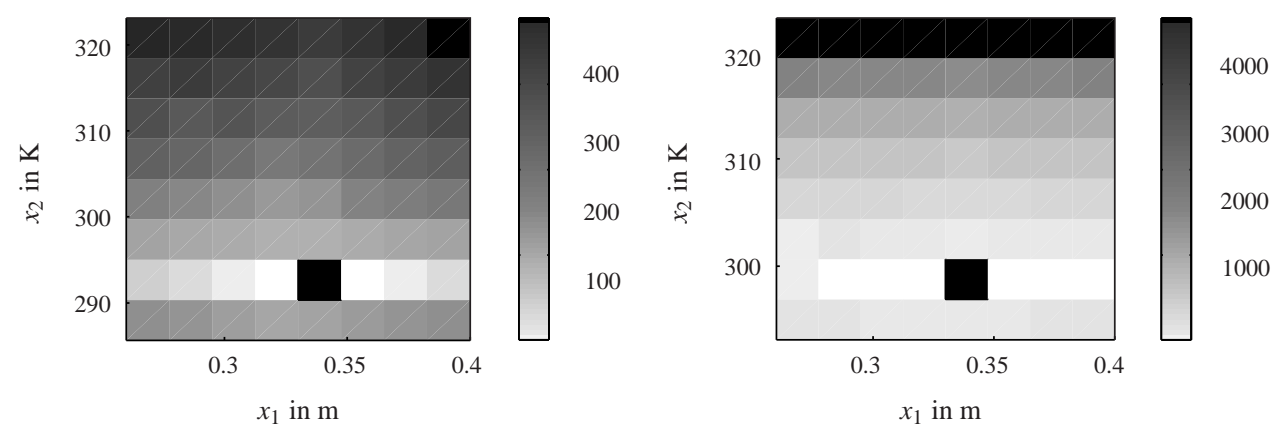

Figure 8: Maximal time in seconds to reach the target set for $\Sigma_{1}$ (left) and $\Sigma_{2}$ (right) over state-space

and the valve angles (33), (34). These settings yield the linearized model of the form (12), (13) for $i=1,2$ with

$$
\begin{array}{rlrl}
\boldsymbol{A}_{1} & =10^{-3}\left(\begin{array}{cc}
-5.74 & 0 \\
-34.5 & -8.58
\end{array}\right), & \boldsymbol{A}_{2} & =10^{-3}\left(\begin{array}{cc}
-5.00 & 0 \\
39.2 & -5.58
\end{array}\right) \\
\boldsymbol{B}_{1} & =10^{-3}\left(\begin{array}{cc}
2.30 & 0 \\
0 & -38.9
\end{array}\right), & \boldsymbol{B}_{2}=10^{-3}\left(\begin{array}{cc}
2.59 & 0 \\
0 & 35.0
\end{array}\right) \\
\boldsymbol{E}_{1}=10^{-3}\left(\begin{array}{c}
0 \\
169
\end{array}\right), & & \boldsymbol{E}_{2}=10^{-3}\left(\begin{array}{c}
1.16 \\
-20.7
\end{array}\right) \\
\boldsymbol{E}_{\mathrm{s} 1}=10^{-3}\left(\begin{array}{cc}
2.42 & 0 \\
43.9 & 5.44
\end{array}\right), & \boldsymbol{E}_{\mathrm{s} 2}=10^{-3}\left(\begin{array}{cc}
2.85 & 0 \\
-46.5 & 5.58
\end{array}\right) \\
\boldsymbol{C}_{\mathrm{z} 1}=\left(\begin{array}{ll}
1 & 0 \\
0 & 1
\end{array}\right), & & \boldsymbol{C}_{\mathrm{z} 2}=\left(\begin{array}{ll}
1 & 0 \\
0 & 1
\end{array}\right)
\end{array}
$$

and the interconnections

$$
\boldsymbol{L}_{12}=\left(\begin{array}{ll}
1 & 0 \\
0 & 1
\end{array}\right), \quad \boldsymbol{L}_{21}=\left(\begin{array}{ll}
1 & 0 \\
0 & 1
\end{array}\right)
$$

The control input generators $C_{1}$ and $C_{2}$ determine the control inputs according to the model (15) using the feedback gains

$$
\boldsymbol{K}_{1}=\left(\begin{array}{cc}
10.5 & 0 \\
0.90 & -0.05
\end{array}\right), \quad \boldsymbol{K}_{2}=\left(\begin{array}{cc}
11.5 & 0 \\
1.10 & 0.40
\end{array}\right) .
$$

These state-feedback gains guarantee the stability of the isolated event-based control loops. In order to prove the stability of the interconnected control loops, the stability test (18) is applied which yields

$$
\lambda_{\mathrm{P}}\left(\int_{0}^{\infty} \overline{\boldsymbol{G}}_{\mathrm{xS}}(t) \overline{\boldsymbol{L}} \overline{\boldsymbol{C}}_{\mathrm{z}} \mathrm{d} t\right)=0.38<1 .
$$

This result implies the boundedness of the state $\boldsymbol{x}(t)$ of the overall event-based control system. According to Theorem 4 the set $\mathcal{B}$ in which the state $\boldsymbol{x}(t)$ is maintained by the local approach can be set by the event threshold vectors of the event generators $E_{1}$ and $E_{2}$ which are chosen to

$$
\overline{\boldsymbol{e}}_{1}=\left(\begin{array}{c}
0.02 \\
0.4
\end{array}\right), \quad \overline{\boldsymbol{e}}_{2}=\left(\begin{array}{c}
0.02 \\
0.4
\end{array}\right) .
$$

Hence, $E_{1}$ and $E_{2}$ trigger an event if either the level or the temperature deviates by $2 \mathrm{~cm}$ or $0.4 \mathrm{~K}$, respectively, from the corresponding model state. According to Eqs. (23)-(25), the choice (40) yields the ultimate bound

$$
\boldsymbol{b}=\left(\begin{array}{llll}
0.018 & 2.56 & 0.027 & 1.36
\end{array}\right)^{\top} .
$$

Consequently, the local approach ensures that the state $\boldsymbol{x}=$

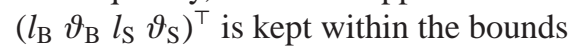

$$
\begin{array}{ll}
l_{\mathrm{B}}(t) \in[0.312 ; 0.348], & \vartheta_{\mathrm{B}}(t) \in[292.1 ; 297.3] \\
l_{\mathrm{S}}(t) \in[0.313 ; 0.367], & \vartheta_{\mathrm{S}}(t) \in[298.8 ; 301.6]
\end{array}
$$

for all $t \geq T\left(x_{0}\right)$ where the time $T\left(x_{0}\right)$ is determined by the global approach. A comparison of the bounds (41) with the desired target set (31) implies that the decentralized event-based controllers with state-feedback gains (39) and the event thresholds (40) satisfy the control aim.

\subsection{Implementation of the event-based controllers}

The event-based controller design methods proposed in Secs. 3 and 4 do not take any technical requirements or restrictions into account. Hence, some of the assumptions that have 
been made for developing the design methods are not satisfied by the technical plant at which the considered continuous flow process is realized. The following summarizes the differences between the theoretical foundations and the actual implementations of the event-based controllers.

State measurement. Both the local and the global approach assume the state $\boldsymbol{x}_{i}(t)$ or the quantized version $\left[\boldsymbol{x}_{i}(t)\right]$, respectively, to be continuously measurable for evaluating the respective control laws, while at the technical plant the state $\boldsymbol{x}_{i}(t)$ is sampled with the sampling time $T_{\mathrm{s}}=0.2 \mathrm{~s}$. This sampling time is by a factor of more than 150 smaller than the time constants of the process and, thus, the error that is introduced by the sampling is assumed to be negligible. A more detailed analysis of event-based control with discrete-time sampling is given in Grüne et al. (2010).

For the practical realization of the event generators $E_{1}$ and $E_{2}$ in the local approach, the event condition (16) is substituted by the condition

$$
\left|\boldsymbol{x}_{\Delta i}(t)\right| \geq \overline{\boldsymbol{e}}_{i}
$$

for $i=1,2$, since the event generators generally only detect the exceeding of the event condition due to the periodic sampling. The event condition of the global approach remains unchanged in the implementation on digital hardware. The global approach uses quantized state information $\left[\boldsymbol{x}_{i}(t)\right]$. In the experimental setup the quantization of the state information is performed in the control algorithm.

Model uncertainties. The nonlinear plant model (1), which is the common basis for both presented approaches, includes uncertainties and, hence, only approximately describes the behavior of the real plant. These model uncertainties are assumed to be negligible.

In the global and the local approach a discretized or a linearized model, respectively, of the nonlinear plant model (1) is used for the controller design. It is a standing assumption that both the discrete-time model (4) as well as the linear model (12), (13) represent the behavior of the plant (1) with sufficient precision in the considered domain of the state space. This assumption will be verified by means of the experiment.

\section{Experimental evaluation}

\subsection{Description of the experiment}

This section presents the results of an experiment where the state $\boldsymbol{x}(t)$ of the system (26), (27) is driven from the initial state

$$
\boldsymbol{x}_{1}(0)=\left(\begin{array}{l}
l_{\mathrm{B}}(0) \\
\vartheta_{\mathrm{B}}(0)
\end{array}\right)=\left(\begin{array}{c}
0.40 \\
317.2
\end{array}\right), \quad \boldsymbol{x}_{2}(0)=\left(\begin{array}{l}
l_{\mathrm{S}}(0) \\
\vartheta_{\mathrm{S}}(0)
\end{array}\right)=\left(\begin{array}{c}
0.40 \\
293.4
\end{array}\right)
$$

to the target set $\mathcal{A}$ as defined in (31) and maintained there. For the transition of the state $\boldsymbol{x}(t)$ to the set $\mathcal{A}$ the system is considered to be undisturbed, whereas the disturbances $\boldsymbol{d}(t)$ are temporarily active while the state $\boldsymbol{x}(t)$ is to be kept within $\mathcal{A}$. The disturbance characteristics are set to

$$
\begin{array}{ll}
d_{\mathrm{H}}(t)=0.1, & \text { for } 800 \leq t \leq 1200, \\
d_{\mathrm{F}}(t)=0.25, & \text { for } 1550 \leq t \leq 1800 .
\end{array}
$$

In the remaining time intervals no disturbance is active.

\subsection{Experimental results}

The behavior of the continuous flow process with decentralized event-based control is illustrated in Figs. 9-12. Figure 9 gives an overview over the transition of the subsystem states $\boldsymbol{x}_{1}(t)$ and $\boldsymbol{x}_{2}(t)$ into the respective target regions. Once, the states enter the target regions, they are kept within these sets which shows that the control aim is fulfilled. Note that this aim is achieved despite model uncertainties which occur, since the model (26), (27) does not precisely describe the behavior of the plant. Hence, this investigation shows that both proposed decentralized event-based control approaches are robust with respect to model uncertainties.

The transition of the state into the target region $\mathcal{A}$ by the global approach is shown in Fig. 10. In reactor B the target region $\mathcal{A}_{1}$ is reached within $T_{1}=398 \mathrm{~s}$, while in reactor $\mathrm{S}$ the state $\boldsymbol{x}_{2}(t)$ enters $\mathcal{A}_{2}$ already after $T_{2}=103 \mathrm{~s}$. The state $\boldsymbol{x}_{2}(t)$ is steered by four times faster to the target region $\mathcal{A}_{2}$ compared to the transition of $\boldsymbol{x}_{1}(t)$ to $\mathcal{A}_{1}$, which is due to the fact that $\boldsymbol{x}_{2}(0)$ is much closer to $\mathcal{A}_{2}$ as $\boldsymbol{x}_{1}(0)$ is to $\mathcal{A}_{1}$. This is also reflected in the number of events triggered in both subsystems: In the reactor S only 5 events are triggered before the local approach is activated, whereas in reactor $\mathrm{B}, 48$ events are generated before the target set $\mathcal{A}_{1}$ is reached.

Figure 11 shows the disturbance rejection behavior of the continuous flow process with the local event-based control approach. The time intervals in which the disturbances $d_{\mathrm{H}}(t)$ and $d_{\mathrm{F}}(t)$ are active are highlighted in gray. The experiments show that in event-based control the feedback communication is adapted to the current system behavior. In the time interval $[103,398] \mathrm{s}$ the state $\boldsymbol{x}_{2}(t)$ is in the target set $\mathcal{A}_{2}$, whereas $\boldsymbol{x}_{1}(t)$ is still outside of $\mathcal{A}_{1}$, which means that reactor $\mathrm{S}$ is considerably affected by reactor $\mathrm{B}$ via the interconnections. In this time interval, 9 events are generated in reactor $\mathrm{S}$ within less than $300 \mathrm{~s}$, while in $[398,1550] \mathrm{s}$, where the coupling effect is
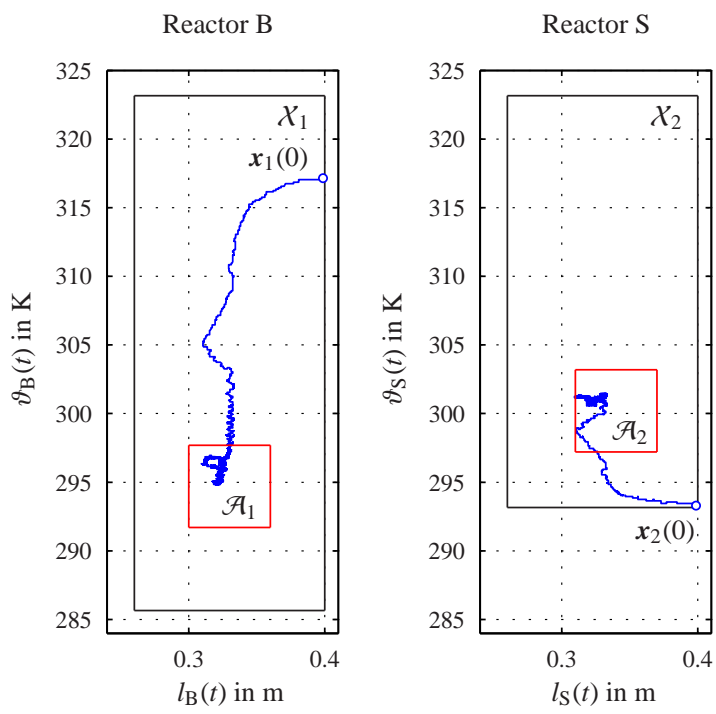

Figure 9: Trajectories of the state $\boldsymbol{x}_{1}(t)$ and $\boldsymbol{x}_{2}(t)$ 

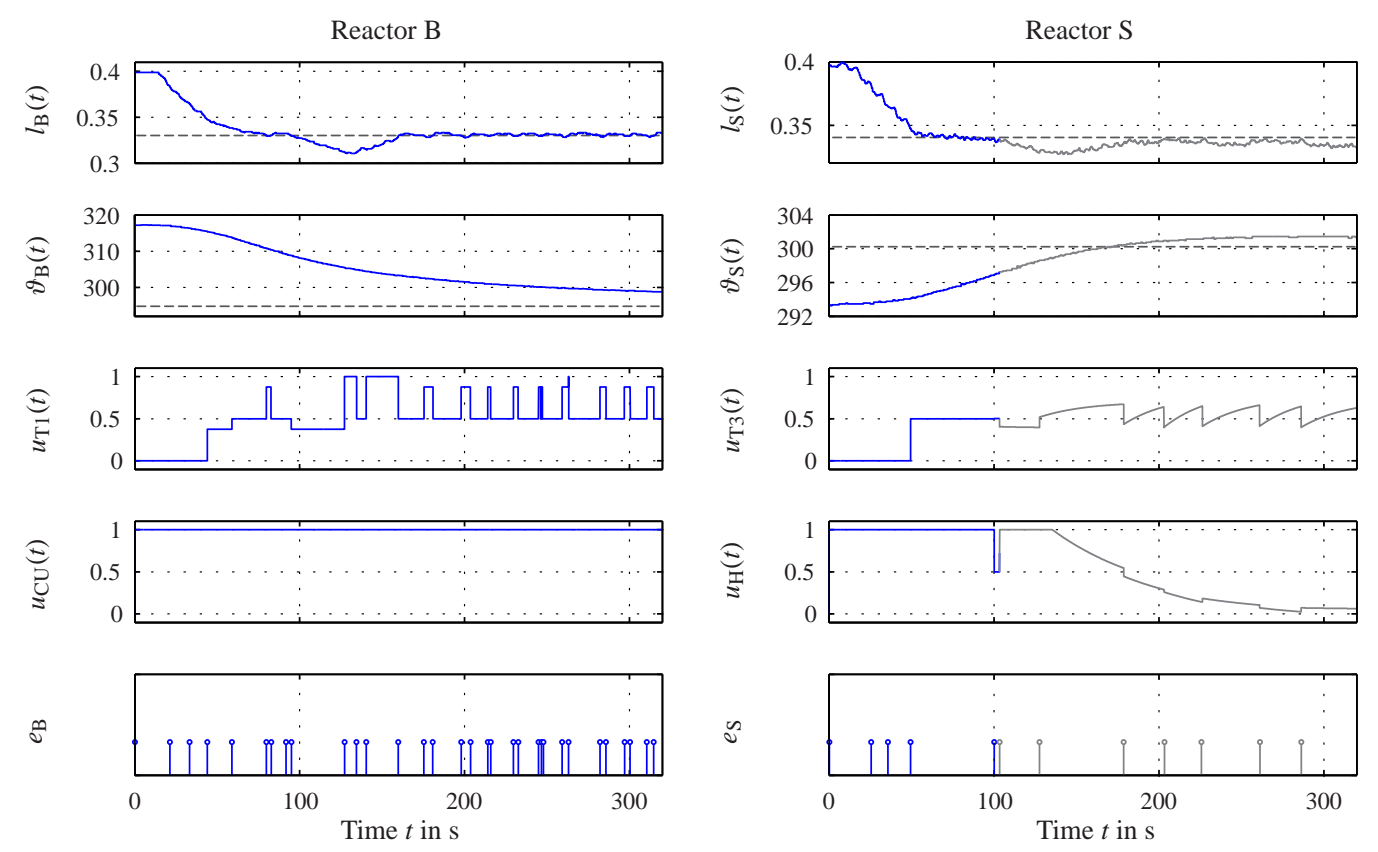

Figure 10: Behavior of the global approach. In the upper two rows the trajectories of the levels $l_{\mathrm{B}}(t), l_{\mathrm{S}}(t)$ and of the temperatures $\vartheta_{\mathrm{B}}(t), \vartheta_{\mathrm{S}}(t)$ are shown for reactor $\mathrm{B}$ and $\mathrm{S}$ on the left-hand side or right-hand side, respectively. The next two rows depict the respective control inputs and the event time instant are marked by stems in the bottom figures.
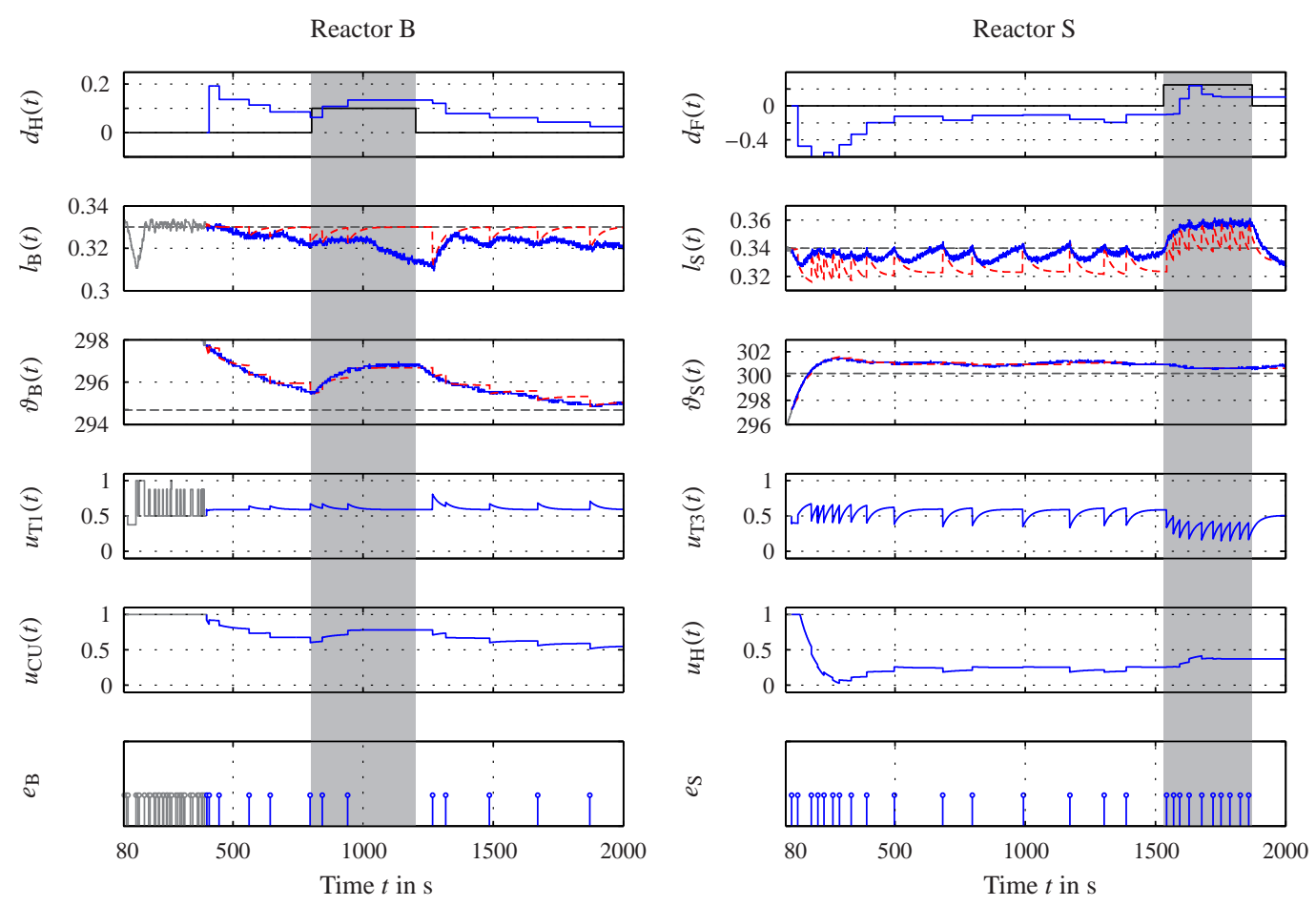

Figure 11: Disturbance rejection behavior of the local approach. The behavior of reactor B and reactor S is depicted in the plots on the left-hand side or right-hand side, respectively. The first row shows the disturbances and the estimated values. The trajectories of the level and temperature are given in the second and third row (solid line: plant state, dashed line: model state). The control inputs are illustrated in the next two rows and the event time instants are represented by stems in the bottom figure.

small and the disturbance $d_{\mathrm{F}}$ is not active, only 7 events are triggered in more than $1150 \mathrm{~s}$. In order to attenuate the disturbance $d_{\mathrm{F}}(t)$ that affects reactor $\mathrm{S}$ in the gray highlighted interval, the feedback communication is induced more often, i. e. 10 events are generated. In total, only 13 events per $1602 \mathrm{~s}$ are triggered in reactor B and 26 events per $1897 \mathrm{~s}$ are triggered in reactor S. Compared to a sampled-data control with a sampling period $T_{\mathrm{s}}=10 \mathrm{~s}$ (which is a typical choice for the considered contin- 
uous flow process), the feedback communication effort is considerably reduced by the event-based control.

Figure 12 provides a verification of the bounds (41) determined according to the analysis method in Theorem 4 . It is shown that in both subsystems, the levels $l_{\mathrm{B}}(t)$ and $l_{\mathrm{S}}(t)$ exceed the calculated bounds, nevertheless, the maximum deviation between the levels and the respective bounds is less than $0.5 \mathrm{~cm}$. From this investigation it can be concluded that the analysis method in Theorem 4 yields tight bounds for the considered class of systems, however, these bounds might not hold in the presence of model uncertainties.

\section{Conclusion}

This paper has presented a new method for the decentralized event-based control of physically interconnected systems and its practical application to a continuous flow process. The proposed control method is based on two approaches, referred to as global and local approach, that differ with respect to their control task. The global event-based control approach uses a nonlinear model of the plant and quantized state information in order to drive the state of each system into a target region. The state is kept within this target set in spite of exogenous disturbances and interconnections to other systems by the local event-based control approach. In this way, both approaches jointly achieve ultimate boundedness of the closed-loop system. The theoretical results have been evaluated on the basis of experimental results, obtained by the application of the control method to a continuous flow process. These results have mainly shown two facts: First, the decentralized event-based control method is robust with respect to model uncertainties and, second, the control aim is accomplished with considerably less feedback communication effort compared to the communication in sampled-data control.
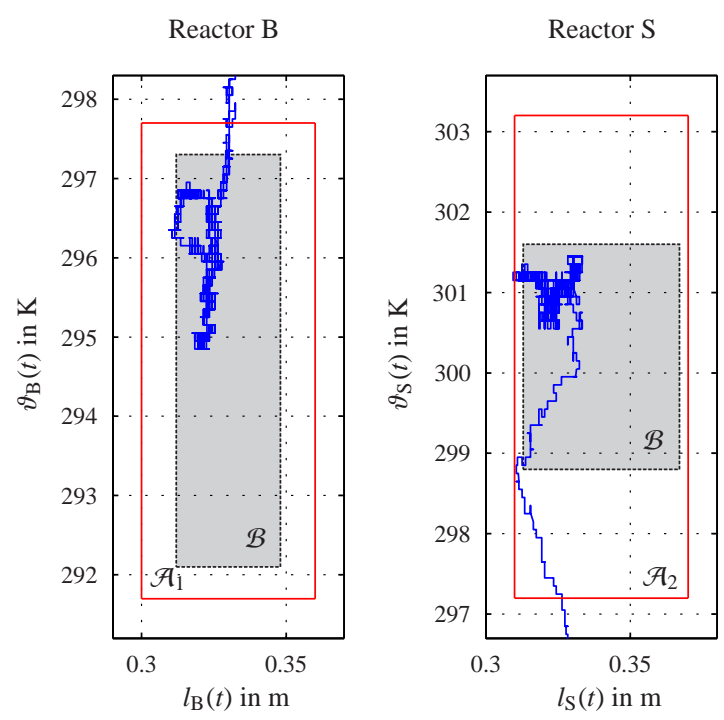

Figure 12: Verification of the ultimate boundedness analysis

\section{Acknowledgements}

This work is supported by the German Research Foundation (DFG) within the priority program "Control Theory of Digitally Networked Dynamical Systems", grant numbers LU 462/262 and GR 1569/11-2 and by the European Union under the 7th Framework Programme FP7-PEOPLE-2010-ITN, Grant agreement nr. 264735-SADCO.

\section{References}

Arzén, K. (1999). A simple event-based PID controller. In Proc. 14th IFAC World Congress (pp. 423-428).

Åström, K. J., \& Bernhardsson, B. (1999). Comparison of periodic and eventbased sampling for first-order stochastic systems. In Proc. 14th IFAC World Congress (pp. 301-306).

Dashkovskiy, S. N., Rüffer, B. S., \& Wirth, F. R. (2010). Small gain theorems for large scale systems and construction of ISS Lyapunov functions. SIAM J. Control Optim., 48, 4089-4118.

De Persis, C., Sailer, R., \& Wirth, F. (2011). On a small-gain approach to distributed event-triggered control. In Proc. 14th IFAC World Congress (pp. 2401-2406).

Donkers, M. C. F., \& Heemels, W. P. M. H. (2012). Output-based eventtriggered control with guaranteed $\mathcal{L}_{\infty}$-gain and improved decentralised event-triggering. IEEE Transactions on Automatic Control, 57, 1362-1376.

Garcia, E., \& Antsaklis, P. J. (2011). Model-based event-triggered control with time-varying network delays. In Proc. Joint IEEE Conference on Decision and Control and European Control Conference (pp. 1650-1655).

Garcia, E., \& Antsaklis, P. J. (2012). Decentralized model-based eventtriggered control of networked systems. In Proc. American Control Conference (pp. 6485-6490).

Gawthrop, P. J., \& Wang, L. B. (2009). Event-driven intermittent control. International Journal of Control, 82, 2235-2248.

Grüne, L., Jerg, S., Junge, O., Lehmann, D., Lunze, J., Müller, F., \& Post, M. (2010). Two complementary approaches to event-based control. Automatisierungstechnik, 58, 173-182.

Grüne, L., \& Junge, O. (2007). Approximately optimal nonlinear stabilization with preservation of the Lyapunov function property. In Proc. IEEE Conference on Decision and Control (pp. 702-707).

Grüne, L., \& Junge, O. (2008). Global optimal control of perturbed systems. $J$. Optim. Theory Appl., 236, 411-429.

Grüne, L., \& Müller, F. (2009). An algorithm for event-based optimal feedback control. In Proc. IEEE Conference on Decision and Control (pp. 5311 5316).

Grüne, L., \& Sigurani, M. (2013). Numerical ISS controller design via a dynamic game approach. In Proc. IEEE Conference on Decision and Control (pp. 1732 - 1737).

Grüne, L., \& Sigurani, M. (2014). A Lyapunov based nonlinear small-gain theorem for discontinuous discrete-time large-scale systems. In Proc. $21 s t$ International Symposium on Mathematical Theory of Networks and Systems (pp. 1031 - 1037).

Heemels, W. P. M. H., Gorter, R. J. A., van den Bosch, P. P. J., Weiland, S., Hendrix, W. H. A., \& Vonder, M. R. (1999). Asynchronous measurement and control: A case study on motor synchronization. Control Engineering Practice, 7, 1467-1482.

Heemels, W. P. M. H., Johansson, K. H., \& Tabuada, P. (2012). An introduction to event-triggered and self-triggered control. In Proc. IEEE Conference on Decision and Control (pp. 3270-3285).

Hendricks, E., Jensen, M., Chevalier, A., \& Vesterholm, T. (1994). Problems in event based engine control. In Proc. American Control Conference (pp. 1585-1587).

Henningsson, T., \& Cervin, A. (2009). Comparison of lti and event-based control for a moving cart with quantized position measurement. In Proc. European Control Conference (pp. 3791-3796).

Henningsson, T., Johannesson, E., \& Cervin, A. (2008). Sporadic event-based control of first-order linear stochastic systems. Automatica, 44, 2890-2895.

Jiang, Z.-P., \& Wang, Y. (2001). Input-to-state stability for discrete-time nonlinear systems. Automatica, 37, 857-869. 
Junge, O., \& Osinga, H. M. (2004). A set oriented approach to global optimal control. ESAIM Control Optim. Calc. Var., 10, 259-270 (electronic).

Khalil, H. K. (2002). Nonlinear Systems. Prentice Hall, Upper Saddle River.

Kwon, W. H., Kim, Y. H., Lee, S. J., \& Paek, K. (1999). Event-based modelling and control for the burnthrough point in sintering processes. IEEE Transactions on Control Systems Technology, 7, 31-41.

Lehmann, D. (2011). Event-Based State-Feedback Control. Logos-Verlag, Berlin.

Lehmann, D., \& Lunze, J. (2011). Extension and experimental evaluation of an event-based state-feedback approach. Control Engineering Practice, 19, 101-112.

Lunze, J. (1992). Feedback Control of Large-Scale Systems. Prentice Hall, London.

Lunze, J., \& Lehmann, D. (2010). A state-feedback approach to event-based control. Automatica, 46, 211-215.

Mazo, M., \& Tabuada, P. (2010). Decentralized event-triggered control over wireless sensor/actuator networks. IEEE Transactions on Automatic Control, 56, 2456-2461.

Molin, A., \& Hirche, S. (2013). On the optimality of certainty equivalence for event-triggered control systems. IEEE Transactions on Automatic Control, $58,470-474$.

Sandee, J. H., Heemels, W. P. M. H., Hulsenboom, S. B. F., \& van den Bosch, P. P. J. (2007). Analysis and experimental validation of a sensor-based eventdriven controller. In Proc. American Control Conference (pp. 2867-2874).

Stöcker, C., \& Lunze, J. (2011). Event-based control of nonlinear systems: An input-output linearization approach. In Proc. Joint IEEE Conference on Decision and Control and European Control Conference (pp. 2541-2546).

Stöcker, C., \& Lunze, J. (2013). Input-to-state stability of event-based statefeedback control. In Proc. European Control Conference (pp. 1145-1150).

Stöcker, C., Vey, D., \& Lunze, J. (2013). Decentralized event-based control: Stability analysis and experimental evaluation. Nonlinear Analysis: Hybrid Systems, 10, 141-155.

Tabuada, P. (2007). Event-triggered real-time scheduling of stabilizing control tasks. IEEE Transactions on Automatic Control, 52, 1680-1685.

Tallapragada, P., \& Chopra, N. (2011). On event triggered trajectory tracking for control affine nonlinear systems. In Proc. Joint IEEE Conference on Decision and Control and European Control Conference (pp. 5377-5382).

Trimpe, S., \& D'Andrea, R. (2011). An experimental demonstration of a distributed and event-based state estimation algorithm. In Proc. 18th IFAC World Congress (pp. 8811-8818).

Wang, X., \& Lemmon, M. D. (2011a). Attentively efficient controllers for event-triggered feedback systems. In Proc. Joint IEEE Conference on Decision and Control and European Control Conference (pp. 4698-4703).

Wang, X., \& Lemmon, M. D. (2011b). Event-triggering in distributed networked control systems. IEEE Transactions on Automatic Control, 56, 586601.

Wang, X., \& Lemmon, M. D. (2012). On event design in event-triggered feedback systems. Automatica, 47, 2319-2322.

Yook, J. K., Tilbury, D. M., \& Soparkar, N. R. (2002). Trading computation for bandwidth: reducing communication in distributed control systems using state estimators. IEEE Transactions on Control Systems Technology, 10, 503-518.

Yu, H., \& Antsaklis, P. J. (2011). Event-triggered real-time scheduling for stabilization of passive and output feedback passive systems. In Proc. American Control Conference (pp. 1674-1679). 